\title{
GEOMETRIC CONSTRUCTIONS ON CYCLES IN $\mathbb{R}^{n}$
}

\author{
BORUT JURČIČ ZLOBEC AND NEŽA MRAMOR KOSTA
}

\begin{abstract}
In Lie sphere geometry, a cycle in $\mathbb{R}^{n}$ is either a point or an oriented sphere or plane of codimension 1 , and it is represented by a point on a projective surface $\Omega \subset \mathbb{P}^{n+2}$. The Lie product, a bilinear form on the space of homogeneous coordinates $\mathbb{R}^{n+3}$, provides an algebraic description of geometric properties of cycles and their mutual position in $\mathbb{R}^{n}$. In this paper, we discuss geometric objects which correspond to the intersection of $\Omega$ with projective subspaces of $\mathbb{P}^{n+2}$. Examples of such objects are spheres and planes of codimension 2 or more, cones and tori. The algebraic framework which Lie geometry provides gives rise to simple and efficient computation of invariants of these objects, their properties and their mutual position in $\mathbb{R}^{n}$.
\end{abstract}

1. Introduction. In his dissertation [10] published in 1872, Sophus Lie introduced his Lie geometry of oriented spheres which is based on a bijective correspondence between oriented geometric cycles, that is, planes and spheres of codimension 1 and points on a quadric surface $\Omega$ in the projective space $\mathbb{P}^{n+2}$. Geometric relations like tangency, angle of intersection, power, etc., are expressed in terms of the Lie product, a nondegenerate bilinear form on the space $\mathbb{R}^{n+3}$ of homogeneous coordinate vectors. Lie geometry is an extension of the perhaps better known Möbius geometry of nonoriented cycles in $\mathbb{R}^{n}$. Both Lie and Möbius geometries provide an algebraic framework for computing geometric invariants of cycles and expressing their mutual position. This makes Lie geometry an appropriate language for dealing with geometric constructions on spheres and planes in $\mathbb{R}^{n}$. It has been used to study a variety of geometric problems on circles and lines in the plane and design algorithms for finding their solutions, for example, in

2010 AMS Mathematics subject classification. Primary 15A63, 51M15, 51M04.

Keywords and phrases. Lie sphere geometry, Lie form, cycles, projective subspace, determinant, projection.

This research was partially supported by the Slovenian Research Agency grant P1-0292 and the ESF project ACAT.

Received by the editors on December 12, 2012, and in revised form on November 22, 2013. 
$[4,12,13]$. In $[6]$, Lie geometry was used to analyze the existence and properties of solutions of geometric constructions associated to the Apollonius construction in $\mathbb{R}^{n}$. In [9], the special case of the Apollonius problem in the plane was considered. In [7], simple algorithms for symbolic solutions of a number of such geometric constructions were given. A thorough treatment of Lie geometry can be found in $[\mathbf{1}, \mathbf{2}]$.

In this paper, we study geometric objects in $\mathbb{R}^{n}$ which are obtained as intersections of projective subspaces of $\mathbb{P}^{n+2}$ with the quadric $\Omega$. For example, the intersection of $\Omega$ with a $(k+1)$-dimensional projective subspace of $\mathbb{P}^{n+2}$ spanned by $k$ cycles and a special element $r \in$ $\mathbb{P}^{n+2}$ determines a $k$-parametric family of geometric cycles in $\mathbb{R}^{n}$. If the spanning cycles correspond to intersecting geometric objects, this determines a subcycle, that is, a sphere or plane of codimension $k$ in $\mathbb{R}^{n}$ which is the common intersection of all geometric cycles belonging to the family. Such a family is known as a Steiner family. Similarly, the intersection of $\Omega$ with a $(k+1)$-dimensional projective subspace spanned by $k$ cycles and a second special element $w \in \mathbb{P}^{n+2}$ determines a $k$-parametric cone family. If the spanning cycles have a common tangent plane, this generates a cone in $\mathbb{R}^{n}$ consisting of all points of tangency of the cycles of the family to the common tangent planes.

Many geometric properties of these objects can be computed from simple, easily computable algebraic invariants of the corresponding projective subspaces. Such an invariant is, for example, the Lie form restricted to the linear subspace of homogeneous coordinate vectors. First of all, its sign determines which projective subspaces have nonempty intersections with $\Omega$ and thus define geometric objects. Second, quotients of determinants, the so-called discriminants, are algebraic invariants of the obtained geometric objects which reflect their geometric properties. Certain projective transformations (that is, linear transformations on the homogeneous coordinates), in particular Lie projections and Lie reflections, enable a simple, easily implementable computation of geometric characteristics.

Following is a description of the main results of this paper. In Section 2, we give a short summary of Lie geometry of oriented geometric cycles where we refer the reader to [6] or (with minor changes in notation) to [2] for the missing proofs and details. 
In Section 3, we introduce general $s$-families, that is, families of cycles which are obtained as intersections of $\Omega$ with a projective subspace of $\mathbb{P}^{n+2}$ containing a distinguished cycle $s$. We then describe our main algebraic tools: determinants, Lie projections and Lie reflections. We focus on hyperbolic families, where the determinant of the corresponding subspace is negative, since these determine geometric objects of interest to us. Depending on a further cycle $s^{\prime}$ we define the $s^{\prime}$ discriminant of a hyperbolic $s$-family. The Lie projection $c$ of $s^{\prime}$ onto the corresponding projective subspace has a special role in the family: it generates the cycle of the family with the minimal $s^{\prime}$-discriminant. We show that the $w$-discriminant of a hyperbolic $r$-family gives the radius of the corresponding subcycle, the projection $c$ determines the cycle in the family with the minimal radius, and the planes of the family correspond to elements in the projection of the dual subspace $\ell=s^{\perp}$. In the case of hyperbolic $w$-families, the $r$-discriminant gives the angle at the vertex of the cone, and $c$ determines the plane, orthogonal to the axis of the cone, while its dual subspace determines the points of the family, in particular, in the case of a 1-parametric cone family, the vertex of the cone.

In Section 4, we consider the mutual position of an $s$-family and a cycle. We define the $s$-discriminant of the two objects and show that its value coincides with the extreme value of the $s$-discriminant on pairs consisting of the given cycle and any cycle from the family and that it is achieved on the Lie projection of the given cycle onto the family. We prove that the $r$-discriminant of a cycle and a subcycle corresponds to the minimal angle of intersection (if it exists) or the maximal angle under which the given cycle is seen from the cycles of the family (if the intersection does not exist), and that this extreme value is achieved on the projection of the given cycle onto the family. On the other hand, the discriminant also gives the angle of the segment connecting the center of the given cycle and a point on the subcycle above the plane in $\mathbb{R}^{n}$ in which the subcycle lies. The $w$-discriminant of a cycle and a cone corresponds to the tangential distance of the given cycle to the cone, which coincides with the minimal tangential distance between cycles of the cone family and the given cycle and is again achieved on the Lie projection of the cycle onto the family.

In Section 5, we generalize this to the case of two families of the same dimension and define their $s$-discriminant. We show that the value of 
the discriminant coincides with the extreme value of the $s$-discriminant on pairs consisting of a cycle from each family, and that it is achieved at the fixed points of a product of two Lie projections.

Finally, we illustrate these results on the case of two subcycles and two cones in $\mathbb{R}^{3}$ : the sign of the $r$-discriminant of two subcycles determines whether they are linked or unlinked, and the fixed point pairs determine the cycles in the two $r$-families with the largest and the smallest angle, while the sign of the $w$-discriminant of a pair of cones determines whether their tangential distance exists, and the fixed point pairs correspond to pairs of cycles from each $w$-family with minimal tangential distance.

All constructions which appear in this paper as well as in $[6,7]$ have been implemented in a Mathematica package which can be found at http://matematika.fe.uni-lj.si/html/people/borut/Lie/. The examples in this paper have all been generated with this package.

2. Cycles. Throughout this paper, we will use the following convention: a lowercase letter will denote an element of a projective space and the corresponding upper case letter its homogeneous coordinate vector.

We start with an informal geometric description of the Möbius and Lie coordinates of cycles in $\mathbb{R}^{n}$. In Möbius geometry, nonoriented cycles in $\mathbb{R}^{n}$ are represented as points on or outside a quadric surface $Q$ in the projective space $\mathbb{P}^{n+1}$ in the following way. The vector space $\mathbb{R}^{n+2}$ of homogeneous coordinate vectors of points in $\mathbb{P}^{n+1}$ is given the Lorentz metric or Möbius product, an indefinite bilinear form of index 1 which we denote by $($,$) . The quadric Q$ consists of points $z \in \mathbb{P}^{n+1}$ such that $(Z, Z)=0$. The quadric $Q$ divides the points of $\mathbb{P}^{n+1}$ into three types: a point $z$ on $Q$ is lightlike, a point $z$ such that $(Z, Z)>0$ is spacelike, and a point $z$ such that $(Z, Z)<0$ is timelike. The cycles of $\mathbb{R}^{n}$ are represented by lightlike and spacelike points of $\mathbb{P}^{n+1}$ by identifying $\mathbb{R}^{n}$ with $\left\{\left(\zeta_{1}, \ldots, \zeta_{n+1}\right) \mid \zeta_{n+1}=0\right\} \subset \mathbb{R}^{n+1} \subset \mathbb{P}^{n+1}$ and $Q$ with the paraboloid

$$
\zeta_{n+1}=-\frac{1}{2} \sum_{i=1}^{n} \zeta_{i}^{2}
$$

tangent to $\mathbb{R}^{n}$ at the origin. 


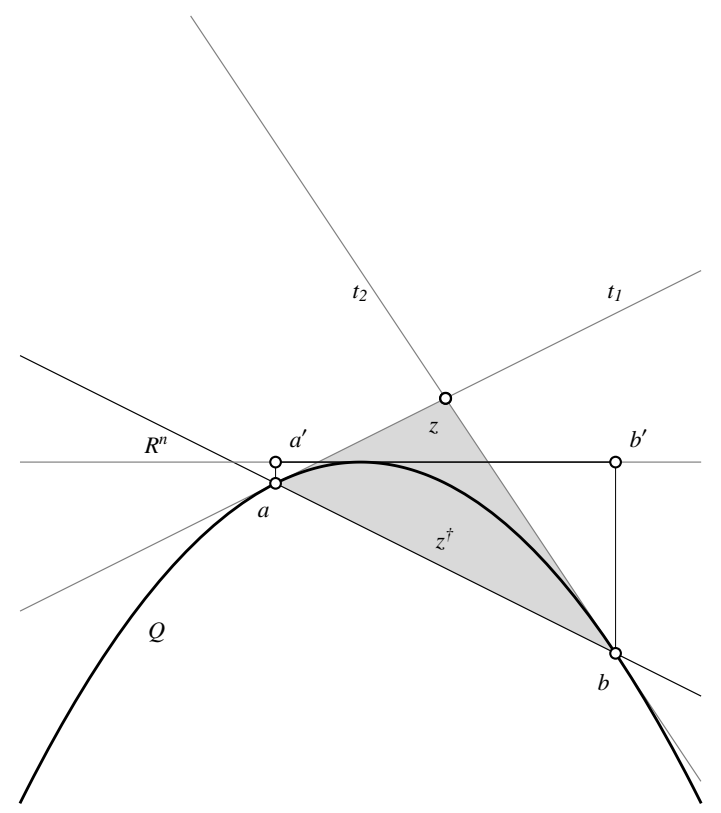

FiguRE 1. In Möbius geometry the 0-dimensional unoriented sphere in $\mathbb{R}^{1}$ consisting of the points $a^{\prime}$ and $b^{\prime}$ is represented by the point $z$ in the plane $\mathbb{R}^{2}$. The 0 -sphere $\left\{a^{\prime}, b^{\prime}\right\}$ is the projection onto $\mathbb{R}^{1}$ of the points $\{a, b\}$ on the Möbius quadric $Q$ and $z$ is the polar point to the line through the points $a$ and $b$.

Each geometric cycle $c$ in $\mathbb{R}^{n}$ can be obtained by intersecting $Q$ with an $n$-plane, $l \subset \mathbb{R}^{n+1}$, and projecting the intersection to $\mathbb{R}^{n}$. If $c$ is a point, that is, a sphere with radius 0 , then $l$ is tangent to $Q$ and $z$ is the point of tangency. If $c$ is a sphere or plane, then $z$ is the polar point of $l$, that is, the common point of all tangent planes to $Q$ at the points of intersection $Q \cap l$, and $z$ is spacelike. If $c$ is a sphere, then $z \in \mathbb{R}^{n+1} \subset \mathbb{P}^{n+1}$ and, if $c$ is a plane, then $z \in \mathbb{P}^{n+1} \backslash \mathbb{R}^{n+1}$. Figure 1 shows this construction in the case of a 0 -dimensional cycle in $\mathbb{R}^{1}$ : a 0 sphere in $\mathbb{R}^{1}$ consisting of two points, denoted by $a^{\prime}$ and $b^{\prime}$, is obtained by projecting the intersection $\{a, b\}$ of the quadric with a line in $\mathbb{R}^{2}$, and $z \in \mathbb{R}^{2} \subset \mathbb{P}^{2}$ is the polar point of this line. 
In Lie geometry, an additional dimension is added, that is, $\mathbb{P}^{n+1}$ is embedded as a projective subspace into $\mathbb{P}^{n+2}$. The Möbius product is extended to the Lie product, an indefinite bilinear form of index 2 on the space of homogeneous coordinate vectors in $\mathbb{R}^{n+3}$. The Lie product of vectors $X, Y \in \mathbb{R}^{n+3}$ will be denoted by $(X \mid Y)$. Each oriented geometric cycle in $\mathbb{R}^{n}$ is represented by a point on the Lie quadric

$$
\Omega=\left\{x \in \mathbb{P}^{n+2} \mid(X \mid X)=0\right\} .
$$

The quadric $\Omega$ is a branched double cover over the spacelike and lightlike points of $\mathbb{P}^{n+1}$ intersecting $\mathbb{P}^{n+1}$ in the branching locus $Q$. A point of $\mathbb{R}^{n}$ is represented by a single point $z \in Q \subset \Omega$, while a sphere or a plane is represented by two points $z^{+}$and $z^{-}$on $\Omega$ lying above and below $z$, one for each orientation. Figure 2 shows the projection from the Lie quadric to the Möbius space.

In Möbius geometry $(X, Y)=0$ if and only if the two geometric cycles represented by $x$ and $y$ intersect orthogonally. More precisely, for any two spacelike points $x, y \in \mathbb{P}^{n+1}$, the Möbius product in suitable homogeneous coordinates gives the angle of intersection of the two cycles they represent.

In Lie geometry the equation $(X \mid Y)=0$ describes oriented contact, that is, $(X \mid Y)=0$ if and only if the two oriented geometric cycles $x, y$ are tangent with compatible orientations in the points of tangency. Because of this, Lie geometry is particularly suited for dealing with configurations of geometric cycles with certain tangency requirements, since such requirements are encoded by the simple linear equation $(X \mid Y)=0$.

In order to give a precise description of the correspondence between oriented geometric cycles and points on $\Omega$ which can be used for specific algorithms and computations, it is necessary to introduce local coordinate charts on $\mathbb{P}^{n+2}$.

We will call an element $x \in \mathbb{P}^{n+2}$ (denoted by a lowercase letter) an algebraic cycle (or, mostly, just a cycle). An algebraic cycle $x$ is given by a nonzero vector of homogeneous coordinates $X \in \mathbb{R}^{n+3}$, which is determined up to a scalar factor and which we denote by the corresponding uppercase letter. 


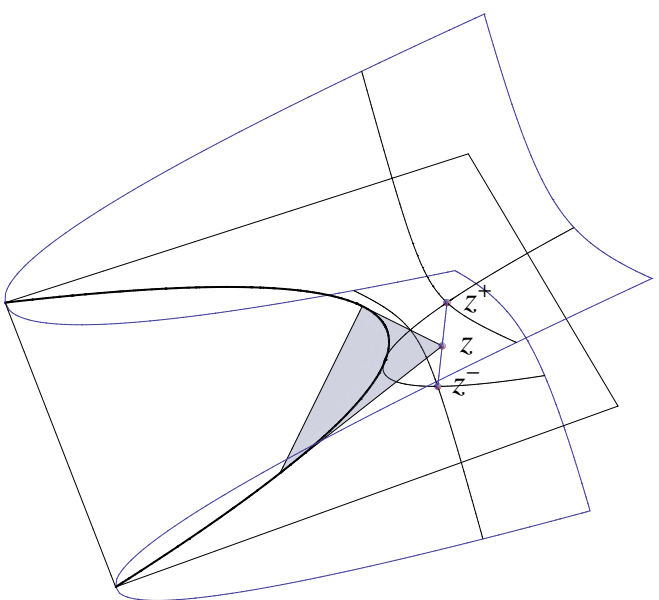

FiguRE 2. In Lie geometry, the points on and outside the Möbius quadric in $\mathbb{P}^{n+1}$ are covered by the Lie quadric $\Omega \subset \mathbb{P}^{n+2}$. A spacelike point $z \in \mathbb{P}^{n+1}$ which represents a nonoriented sphere in Möbius geometry is covered by the two points $z^{-}, z^{+} \in \mathbb{P}^{n+2}$ representing the sphere with both orientations.

The Lie product on $\mathbb{R}^{n+3}$ is a nondegenerate bilinear form of index 2 given by

$$
(X \mid Y)=X^{T} \mathbf{A} Y, \quad \mathbf{A}=\left[\begin{array}{cccc}
0 & \mathbf{0} & 1 & 0 \\
\mathbf{0} & \mathbf{I}^{n} & \mathbf{0} & \mathbf{0} \\
1 & \mathbf{0} & 0 & 0 \\
0 & \mathbf{0} & 0 & -1
\end{array}\right]
$$

where $\mathbf{I}^{n}$ denotes the $n \times n$ identity matrix. In coordinates, let $X=\left(\xi_{0}, \boldsymbol{\xi}_{1}, \xi_{2}, \xi_{3}\right)$ and $Y=\left(\eta_{0}, \boldsymbol{\eta}_{1}, \eta_{2}, \eta_{3}\right)$, where $\xi_{i}, \eta_{i} \in \mathbb{R}$ for $i=0,2,3$ and $\boldsymbol{\xi}_{1}, \boldsymbol{\eta}_{1} \in \mathbb{R}^{n}$. Then

$$
(X \mid Y)=\xi_{0} \eta_{2}+\boldsymbol{\xi}_{1} \cdot \boldsymbol{\eta}_{1}+\xi_{2} \eta_{0}-\xi_{3} \eta_{3} .
$$


The vectors $X$ such that $(X \mid X)=0$ form the Lie quadric

$$
\Omega:=\left\{x \in \mathbb{P}^{n+2} \mid(X \mid X)=0\right\} \subset \mathbb{P}^{n+2} .
$$

Cycles $x \in \Omega$ will be called proper cycles, while cycles $x \notin \Omega$ will be called nonproper cycles.

If $\mathbf{X}=\left(X_{1}, \ldots, X_{k}\right)$ denotes a list of homogeneous coordinate vectors, the symbol $\langle\mathbf{X}\rangle=\left\langle X_{1}, \ldots, X_{k}\right\rangle$ will stand for the linear subspace spanned by the vectors $\left(X_{1}, \ldots, X_{k}\right) \in \mathbb{R}^{n+3}$, and the symbol $\langle\mathbf{X}\rangle^{\perp}=\left\langle X_{1}, \ldots, X_{k}\right\rangle^{\perp}$ for the orthogonal complement to $\langle\mathbf{X}\rangle$ with respect to the Lie product, i.e.,

$$
\langle\mathbf{X}\rangle^{\perp}=\left\{Y \mid\left(X_{i} \mid Y\right)=0, i=1, \ldots, k\right\} .
$$

Following our convention on upper and lowercase letters, $\langle\mathbf{x}\rangle=$ $\left\langle x_{1}, \ldots, x_{k}\right\rangle$ and $\langle\mathbf{x}\rangle^{\perp}=\left\langle x_{1}, \ldots, x_{k}\right\rangle^{\perp}$ will denote the projective subspace spanned by $\mathbf{x}$ and its dual projective subspace, respectively. For any nonzero vector $S \in \mathbb{R}^{n+3}$, the open set $\mathcal{U}_{s}=\mathbb{P}^{n+2} \backslash\langle s\rangle^{\perp}$ together with the map

$$
\varphi_{S}: \mathcal{U}_{s} \longrightarrow \mathbb{R}^{n+3}, \quad \varphi_{S}(x):=\frac{1}{(X \mid S)} X,
$$

where $X$ is any vector of homogeneous coordinates of $x$, with image in $\{X \mid(X \mid S)=1\} \cong \mathbb{R}^{n+2}$, is a chart on $\mathbb{P}^{n+2}$ specifying local coordinates in $\mathcal{U}_{s}$. The collection

$$
\left\{\left(\mathcal{U}_{s}, \varphi_{S}\right) \mid S \neq 0 \in \mathbb{R}^{n+3}\right\}
$$

gives the standard manifold structure on $\mathbb{P}^{n+2}$.

Two cycles and their corresponding charts have a special role in Lie geometry: the nonproper cycle $r$ with homogeneous coordinates $R=(0, \mathbf{0}, 0,1)$ and the proper cycle $w$ with homogeneous coordinates $W=(1, \mathbf{0}, 0,0)$. The reason for this is that the equation $(X \mid X)=0$ implies that either $\xi_{2} \neq 0$ and $X \in \mathcal{U}_{w}$, or $\xi_{3} \neq 0$ and $X \in \mathcal{U}_{r}$, so

$$
\Omega \subset \mathcal{U}_{w} \cup \mathcal{U}_{r}
$$

An oriented geometric cycle in $\mathbb{R}^{n}$ is represented by a proper algebraic cycle $x \in \Omega \subset \mathbb{P}^{n+2}$ in the following way.

- The positively oriented (i.e., inward normal) and negatively oriented sphere with center $\mathbf{p}$ and radius $\rho$ are represented by 
the cycles $x$ and $x^{\prime}$ in $\mathcal{U}_{w} \cap \mathcal{U}_{r}$ with local coordinates

$$
\begin{gathered}
\varphi_{W}(x)=(\nu, \mathbf{p}, 1, \rho), \quad \varphi_{R}(x)=\left(-\frac{\nu}{\rho},-\frac{\mathbf{p}}{\rho},-\frac{1}{\rho},-1\right) \\
\varphi_{W}\left(x^{\prime}\right)=(\nu, \mathbf{p}, 1,-\rho), \quad \varphi_{R}\left(x^{\prime}\right)=\left(\frac{\nu}{\rho}, \frac{\mathbf{p}}{\rho}, \frac{1}{\rho},-1\right),
\end{gathered}
$$

respectively, where $\nu=\left(\rho^{2}-\|\mathbf{p}\|^{2}\right) / 2$ and $\rho>0$.

- A point $\mathbf{p} \in \mathbb{R}^{n}$, i.e., a sphere with radius 0 , is represented by the cycle $x \in\langle r\rangle^{\perp} \subset \mathcal{U}_{w}$ with local coordinates $\varphi_{W}(x)=$ $(\nu, \mathbf{p}, 1,0)$, where $\nu=-\|p\|^{2} / 2$.

- A plane with normal $\mathbf{n}$, where $\|\mathbf{n}\|=1$, containing the point $\mathbf{q}$ is represented by the cycle $x \in\langle w\rangle^{\perp} \cap U_{r}$ with local coordinates

$$
\varphi_{R}(x)=(\mathbf{n} \cdot \mathbf{q},-\mathbf{n}, 0,-1) .
$$

In the opposite direction, every proper cycle $x \neq w$ represents an oriented geometric cycle in $\mathbb{R}^{n}$. A proper cycle in $\langle w\rangle^{\perp} \subset \mathcal{U}_{r}$ represents a plane, while a proper cycle in $\langle r\rangle^{\perp} \subset \mathcal{U}_{w}$ represents a point in $\mathbb{R}^{n}$. The only exception is $w \in\langle w\rangle^{\perp} \cap\langle r\rangle^{\perp}$, which does not represent any geometric cycle. A change of sign of the last homogeneous coordinate of a cycle $x \in \Omega$, produces the reoriented cycle $x^{\prime} \in \Omega$ representing the same geometric cycle and an oriented cycle with the opposite orientation. Points have no orientation: if $x$ is a point, then $x^{\prime}=x$.

Remark 2.1. Spheres and planes in $\mathbb{R}^{n}$ correspond through the stereographic projection to codimension 1 spheres on the sphere $S^{n}$ and, in this setting, the cycle $w$ is the representation of the pole in $S^{n}$.

The Lie product computed in different charts reflects different geometric properties of the corresponding pair of cycles. Following are some specific cases.

(1) Let $x_{1}$ and $x_{2}$ be proper cycles representing geometric cycles $c_{1}$ and $c_{2}$ such that $\left(X_{1} \mid X_{2}\right)=0$. If one of the cycles, for example $c_{1}$, is a point, then it lies on $c_{2}$. If both are non-point cycles, then $c_{1}$ and $c_{2}$ are tangent with compatible orientation. If $c_{1}$ and $c_{2}$ are both planes, then they are parallel with compatible orientation. The proof of this fact amounts to simple geometric verifications and can be found for example in [2]. 
(2) Let $x_{1}, x_{2} \in \mathcal{U}_{r} \cap \Omega$ be two non-point cycles representing intersecting geometric cycles $c_{1}$ and $c_{2}$. The Lie product is

$$
\left(\varphi_{R}\left(x_{1}\right) \mid \varphi_{R}\left(x_{2}\right)\right)=-\cos \alpha-1,
$$

where $\alpha$ is the angle of intersection (Figure 3, left). If both cycles are spheres, this follows from the law of cosines since

$$
\begin{aligned}
\left(\varphi_{R}\left(x_{1}\right) \mid \varphi_{R}\left(x_{2}\right)\right) & =\frac{-\left\|\mathbf{p}_{1}-\mathbf{p}_{2}\right\|^{2}+\rho_{1}^{2}+\rho_{2}^{2}-2 \rho_{1} \rho_{2}}{2 \rho_{1} \rho_{2}} \\
& =-\cos \alpha-1 .
\end{aligned}
$$

If one cycle is a plane and one is a sphere, then

$$
\begin{aligned}
\left(\varphi_{R}\left(x_{1}\right) \mid \varphi_{R}\left(x_{2}\right)\right) & =\left(\varphi_{R}\left(x_{1}\right) \mid \frac{\varphi_{W}\left(x_{2}\right)}{\rho_{2}}\right) \\
& =\frac{\mathbf{n}_{1} \cdot\left(\mathbf{p}_{2}-\mathbf{q}_{1}\right)-\rho_{2}}{\rho_{2}}=-\cos \alpha-1,
\end{aligned}
$$

where $\mathbf{q}_{1}$ is a point in the intersection. And, finally, if both cycles are planes, again,

$$
\left(\varphi_{R}\left(x_{1}\right) \mid \varphi_{R}\left(x_{2}\right)\right)=\mathbf{n}_{1} \mathbf{n}_{2}-1=-\cos \alpha-1 .
$$

If the cycles $c_{1}$ and $c_{2}$ do not intersect, the Lie product is associated with the Lorentz boost $\chi$.

$\left(\phi_{R}\left(x_{1}\right) \mid \phi_{R}\left(x_{2}\right)\right)= \pm \cosh \chi-1$ and $\cosh \chi=\left|\frac{\rho_{1}^{\prime 2}+\rho_{2}^{\prime 2}}{2 \rho_{1}^{\prime} \rho_{2}^{\prime}}\right|$,

where $\rho_{1}^{\prime}$ and $\rho_{2}^{\prime}$ are the radii of the corresponding concentric cycles (see Figure 3 right) with the same product (2.5).

(3) Let $x_{1}, x_{2} \in \mathcal{U}_{w} \cap \Omega$ be non-point cycles representing geometric cycles $c_{1}$ and $c_{2}$ with a common tangent plane, i.e., with $\left(\rho_{1}-\rho_{2}\right)^{2}-\left\|\mathbf{p}_{1}-\mathbf{p}_{2}\right\|^{2} \leq 0$ (where $\rho_{i}$ can be negative, depending on the orientation). Then

$$
\left(\varphi_{W}\left(x_{1}\right) \mid \varphi_{W}\left(x_{2}\right)\right)=\frac{\left(\rho_{1}-\rho_{2}\right)^{2}-\left\|\mathbf{p}_{1}-\mathbf{p}_{2}\right\|^{2}}{2}=-\frac{d^{2}}{2},
$$

where $d$ is the tangential distance (Figure 4, left).

If the cycles do not have a common tangent plane, then the product (2.6) is positive. If $c_{1}$ is a point, then it lies inside the 

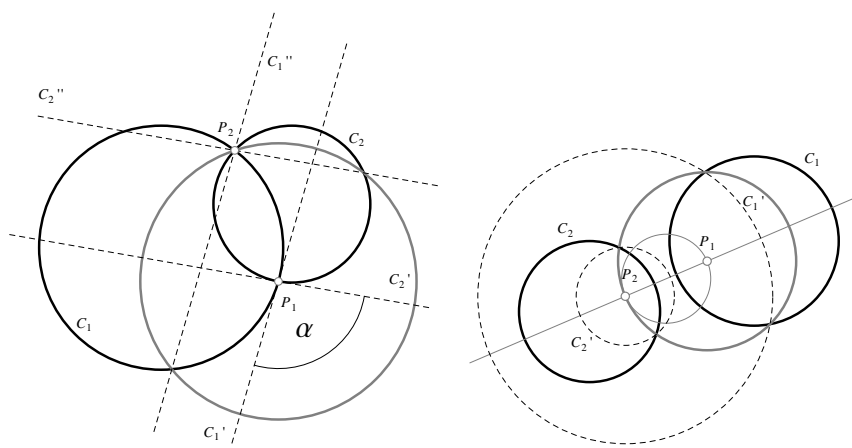

FIGURE 3. Geometric interpretation of the Lie product in the chart $\mathcal{U}_{r}$ in the case of intersecting (left, equation (2.4)), and nonintersecting (right, equation (2.5)) cycles.
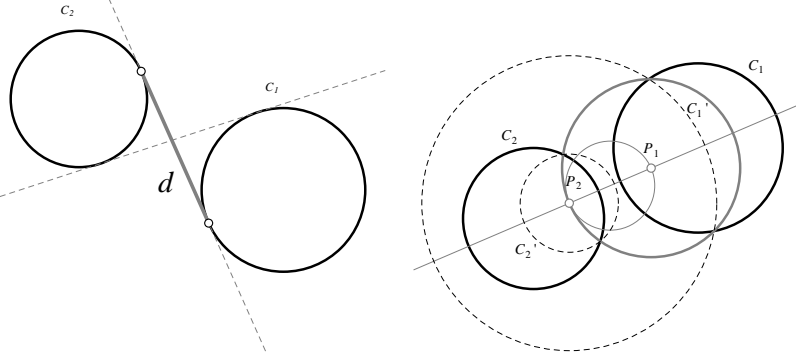

FIGURE 4. Geometric interpretation of the Lie product in the chart $\mathcal{U}_{w}$. On the left, the two cycles have a common tangent plane (equation (2.6)) and have opposite orientations so $\left(\rho_{1}-\rho_{2}\right)$ corresponds to the sum of the two radii and $d$ is the tangential distance. On the right, the cycles do not have a common tangent plane (equation (2.7)) and $d$ is the half chord.

sphere $c_{2}$ and equation (2.6) is

$$
\left(\varphi_{W}\left(x_{1}\right) \mid \varphi_{W}\left(x_{2}\right)\right)=\frac{\rho_{2}^{2}-\left\|\mathbf{p}_{1}-\mathbf{p}_{2}\right\|^{2}}{2}=\frac{d^{2}}{2},
$$

where $d$ is the half chord of $c_{2}$ through $c_{1}$. If both $c_{1}$ and $c_{2}$ 
are spheres, then equation (2.6) gives

$$
\left(\varphi_{W}\left(x_{1}\right) \mid \varphi_{W}\left(x_{2}\right)\right)=\frac{\left(\rho_{1}-\rho_{2}\right)^{2}-\left\|\mathbf{p}_{1}-\mathbf{p}_{2}\right\|^{2}}{2}=\frac{d^{2}}{2},
$$

where $d$ is the half chord of the circle concentric to $c_{2}$ with radius $\left|\rho_{1}\right|+\left|\rho_{2}\right|$ through the center of $c_{1}$. A proof of this is a nice application of Lie reflections and is given later in Corollary 3.6.

\section{Projective subspaces and families of cycles.}

3.1. Families and s-families of cycles. In this section, we will consider families of proper cycles arising from projective subspaces in $\mathbb{P}^{n+2}$ and geometric objects corresponding to them.

Let $\mathbf{x}=\left(x_{1}, \ldots, x_{k+1}\right), 2 \leq k \leq n$, denote a list of cycles spanning the subspace $\langle\mathbf{x}\rangle \subset \mathbb{P}^{n+2}$. If the intersection $\langle\mathbf{x}\rangle \cap \Omega$ is nonempty, it is a family of proper cycles. The dual algebraic object $\langle\mathbf{x}\rangle^{\perp} \cap \Omega$ will be called the cofamily. On the geometric side, the cofamily contains all oriented geometric cycles which are tangent to geometric cycles corresponding to all $x \in \mathbf{x}$.

Typically, we will consider lists of the type $(\mathbf{x}, s)=\left(x_{1}, \ldots, x_{k}, s\right)$, where $x_{i}$ are proper cycles and $s$ is $r, w$ or possibly some other special cycle with with $(S \mid S) \leq 0$. A family $\langle\mathbf{x}, s\rangle$ of this type will be called an $s$-family and the corresponding cofamily will be an s-cofamily. For example, an $r$-cofamily consists of points, and a $w$-cofamily consists of planes. If $k=2$, an $s$-family is usually called a pencil of cycles. Following the standard terminology for pencils, we will call an $r$-family a Steiner family and a $w$-family a cone family.

Let us take a closer look at geometric pencils in $\mathbb{R}^{3}$ (see also $[\mathbf{6}, \mathbf{1 3}]$ ). A Steiner pencil is determined by two intersecting cycles and consists of all cycles containing the intersection circle or line of these two cycles, so the geometric object it represents is the intersecting circle. The cycles of the copencil are the points of this circle. A cone pencil is determined by two oriented spheres with a common tangent plane. The cycles of the copencil are the planes tangent (with compatible orientations) to both spheres. The envelope of these planes is a double cone tangent to all the spheres in the pencil with vertex in the common intersection point of the tangent planes, so the geometric object represented by a 
cone family is an oriented double cone (which collapses to a line if both spanning cycles are points, or extends to a cylinder if the two spanning cycles are spheres with equal radii). Another interesting geometric pencil is obtained if the special cycle $s$ is given by $S=\rho W+R$. Then an $s$-pencil spanned by two oriented spheres consists of all spheres with a common tangent sphere of radius $\rho$. The cycles of the copencil are all spheres of radius $\rho$, tangent to both given spheres. The envelope of these spheres forms a torus, so the new geometric object represented by such a pencil is an oriented torus or, if the two spanning cycles are planes, a cylinder.

3.2. The determinant of a family. Consider the Gram matrix with elements the Lie products of vectors from a list of linearly independent vectors $\mathbf{X}=\left(X_{1}, \ldots, X_{k}\right)$,

$$
\mathbf{A}_{\mathbf{X}}=[\mathbf{X}]^{T} \mathbf{A}[\mathbf{X}]=\left[\begin{array}{ccc}
\left(X_{1} \mid X_{1}\right) & \cdots & \left(X_{1} \mid X_{k}\right) \\
\vdots & \ddots & \vdots \\
\left(X_{k} \mid X_{1}\right) & \cdots & \left(X_{k} \mid X_{k}\right)
\end{array}\right]
$$

Its determinant $\Delta(\mathbf{X})=\operatorname{det} \mathbf{A}_{\mathbf{X}}$ can be positive, negative or even zero, due to the indefiniteness of the Lie product. The sign of the determinant $\Delta(\mathbf{X})$ is an invariant of the projective subspace $\langle\mathbf{x}\rangle$ and, consequently, an invariant of the underlying family $\langle\mathbf{x}\rangle \cap \Omega$. It depends on the index of the Lie form restricted to the subspace $\langle\mathbf{X}\rangle$. The following proposition is a standard result in linear algebra; for example, it is an immediate consequence of [2, Theorem 1.2].

\section{Proposition 3.1.}

(i) If the Lie form on the subspace $\langle\mathbf{X}\rangle$ is nondegenerate, then it is nondegenerate also on the Lie orthogonal complement $\langle\mathbf{X}\rangle^{\perp}$, and $\mathbb{R}^{n+3}=\langle\mathbf{X}\rangle \oplus\langle\mathbf{X}\rangle^{\perp}$.

(ii) If $\Delta(\mathbf{X})<0$, then the index of the Lie form on the subspace $\langle\mathbf{X}\rangle$ is 1 .

The sign of $\Delta(\mathbf{X})$ determines the position of the projective subspace $\langle\mathbf{x}\rangle$, and its Lie orthogonal complement with respect to the Lie quadric $\Omega$, as the following theorem shows.

Theorem 3.2. Let $\mathbf{X}=\left(X_{1}, \ldots X_{k}\right)$, where $2 \leq k \leq n+1$ are linearly independent vectors from $\mathbb{R}^{n+3}$. 
1722 BORUT JURČIČ ZLOBEC AND NEŽA MRAMOR KOSTA

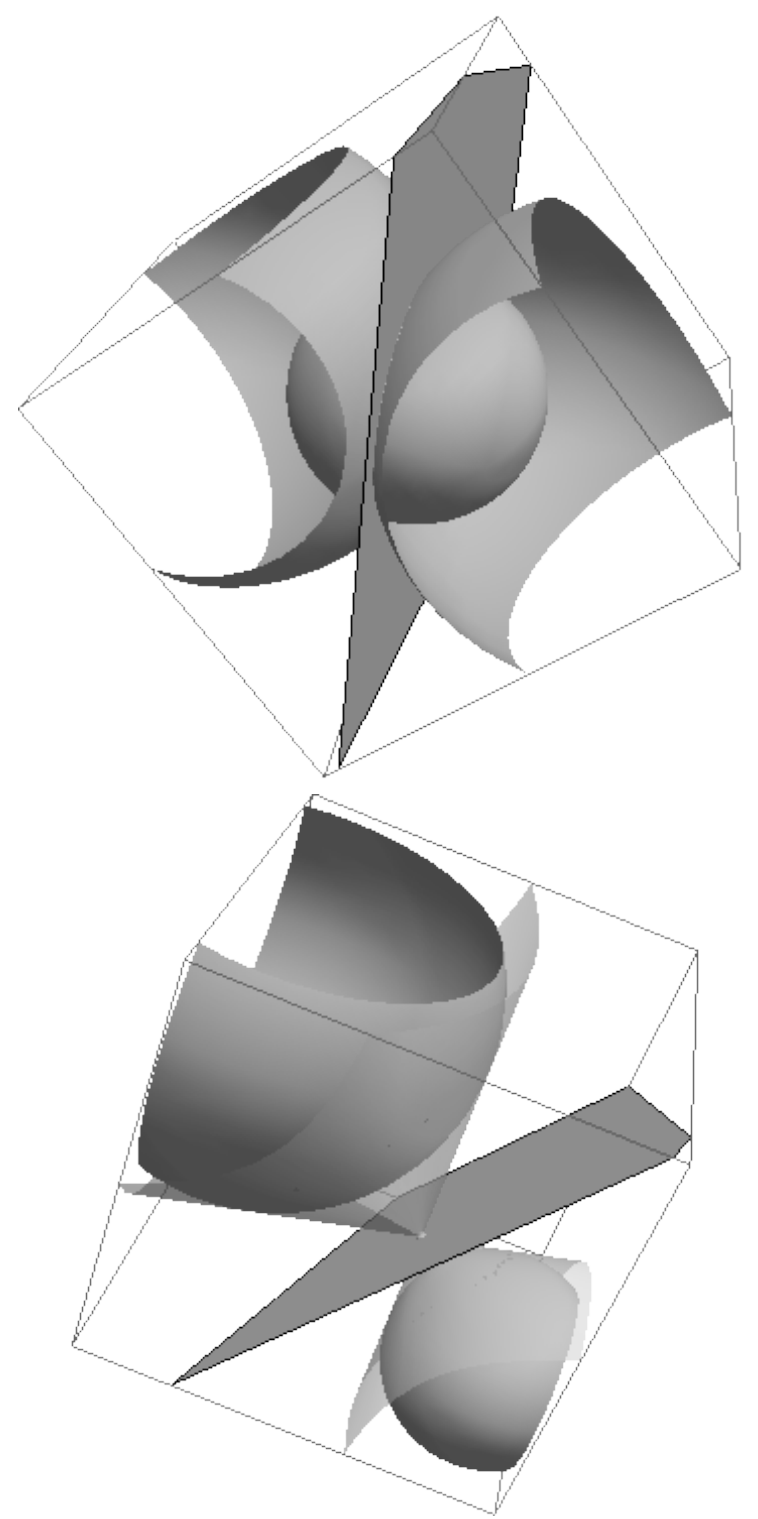

FiguRE 5. A Steiner and a cone pencil in three dimensions. 
(i) If $\Delta(\mathbf{X})<0$, then both $\langle\mathbf{x}\rangle$ and its Lie orthogonal complement $\langle\mathbf{x}\rangle^{\perp}$ intersect $\Omega$.

(ii) If $\Delta(\mathbf{X})=0$, then $\langle\mathbf{x}\rangle \cap\langle\mathbf{x}\rangle^{\perp} \neq \emptyset \subset \Omega$.

(iii) If $\Delta(\mathbf{X})>0$, then exactly one of $\langle\mathbf{x}\rangle$ and $\langle\mathbf{x}\rangle^{\perp}$ does not intersect $\Omega$.

\section{Proof.}

(i) If $\Delta(\mathbf{X})<0$, the restriction of the Lie form to $\langle\mathbf{X}\rangle$, is nondegenerate and has index 1 , so there exists a Lie orthogonal basis of $\langle\mathbf{X}\rangle$ formed by vectors $Y_{i}$ such that $\left(Y_{i} \mid Y_{i}\right)>0, i=1, \ldots, k-1$ and $\left(Y_{k} \mid Y_{k}\right)<0$, (compare [2, Theorem 1.2]). So the projective line $\left\langle y_{i}, y_{k}\right\rangle, 1 \leq i<k$, intersects the quadric $\Omega$. The same is true for Lie orthogonal complement $\langle\mathbf{X}\rangle^{\perp}$.

(ii) If $\Delta(\mathbf{X})=0$, the Lie form is degenerate on the subspace $\langle\mathbf{X}\rangle$, and there exists a vector $X$ Lie orthogonal to all vectors of the subspace including itself, so $x \in\langle\mathbf{x}\rangle^{\perp} \cap\langle\mathbf{x}\rangle \subset \Omega$.

(iii) Finally, in the case $\Delta(\mathbf{X})>0$, we have two possibilities. Either the index of the Lie form is 2 on $\langle\mathbf{X}\rangle$ and 0 on $\langle\mathbf{X}\rangle^{\perp}$ and $\langle\mathbf{X}\rangle$ intersects $\Omega$ while $\langle\mathbf{X}\rangle^{\perp}$ does not, or the other way around.

An immediate application of Theorem 3.2 is a necessary and sufficient condition for the existence of solutions of the oriented Apollonius problem in $\mathbb{R}^{n}$ which asks for an oriented geometric cycle tangent (with compatible orientations) to $n+1$ given oriented cycles $\mathbf{c}=\left(c_{1}, \ldots, c_{n+1}\right)$.

Corollary 3.3. The oriented Apollonius problem on a configuration of $n+1$ oriented cycles $\mathbf{c}$ with corresponding algebraic cycles $\mathbf{x}$, which are represented by the linearly independent set of homogeneous coordinate vectors $\mathbf{X}$, has exactly two solutions if $\Delta(\mathbf{X})<0$, no solution if $\Delta(\mathbf{X})>0$, and one solution if $\Delta(\mathbf{X})=0$.

Proof. A solution of the Apollonius problem is given by an intersection of $\langle\mathbf{x}\rangle^{\perp}$ with the Lie quadric. If $\Delta(\mathbf{X})<0$, then $\langle\mathbf{x}\rangle^{\perp}$ is a projective line which intersects the Lie quadric in two cycles corresponding to the two solutions. If $\Delta(\mathbf{X})>0$, then, since $\langle\mathbf{x}\rangle$ intersects the quadric its dual $\langle\mathbf{x}\rangle^{\perp}$ does not, and the problem has no solutions. Finally, if $\Delta(\mathbf{X})=0$, then the projective line $\langle\mathbf{x}\rangle^{\perp}$ is tangent to $\Omega$, so it 
contains exactly one point from $\Omega$; therefore, the problem has exactly one solution.

3.3. Lie projections and Lie reflections. Let $\mathbf{X}=\left(X_{1}, \ldots, X_{k}\right) \in$ $\mathbb{R}^{n+3}$ be a $k$-tuple of linearly independent vectors such that $\Delta(\mathbf{X}) \neq 0$ (that is, with $\mathbf{A}_{\mathbf{X}}$ nonsingular). The Lie orthogonal projection onto the subspace $\langle\mathbf{X}\rangle$ is given by

$$
P_{\langle\mathbf{X}\rangle} Y=[\mathbf{X}] \mathbf{A}_{\mathbf{X}}^{-1}[\mathbf{X}]^{T} \mathbf{A} Y .
$$

The Lie orthogonal projection onto $\langle\mathbf{X}\rangle$ determines a projective map

$$
P_{\langle\mathbf{x}\rangle}: \mathbb{P}^{n+2} \backslash\langle\mathbf{x}\rangle^{\perp} \rightarrow \mathbf{x} \subset \mathbb{P}^{n+2} .
$$

Proposition 3.4. The Lie orthogonal projection has the following properties:

(i) $P_{\langle\mathbf{X}\rangle^{\perp}}=\mathrm{Id}-P_{\langle\mathbf{X}\rangle}$.

(ii) For any $Y_{1}, Y_{2}, Y \in \mathbb{R}^{n+3}$,

$$
\left(Y_{1} \mid P_{\langle\mathbf{X}\rangle} Y_{2}\right)=\left(P_{\langle\mathbf{X}\rangle} Y_{1} \mid Y_{2}\right)
$$

and

$$
\left(Y \mid P_{\langle\mathbf{X}\rangle} Y\right)=\left(P_{\langle\mathbf{X}\rangle} Y \mid P_{\langle\mathbf{X}\rangle} Y\right)
$$

Proof.

(i) Since $\Delta(\mathbf{X}) \neq 0$, the Lie form is nondegenerate on $\mathbf{X}$ and, by Lemma 3.1, each vector $Y \in \mathbb{R}^{n+3}$ has a unique decomposition into $Y=Y_{1}+Y_{2}$, with $Y_{1} \in\langle\mathbf{X}\rangle$ and $Y_{2} \in\langle\mathbf{X}\rangle^{\perp}$. Then $P_{\langle\mathbf{X}\rangle}(Y)=$ $Y_{1}$ and $P_{\langle\mathbf{X}\rangle^{\perp}}(Y)=Y_{2}$; and so, $P_{\langle\mathbf{X}\rangle}(Y)=Y-P_{\langle\mathbf{X}\rangle}(Y)$.

(ii) Since $\mathbf{A}$, as well as $\mathbf{A}_{\mathbf{X}}=\left([\mathbf{X}]^{T} \mathbf{A}[\mathbf{X}]\right)$, is symmetric

$$
\begin{aligned}
\left(Y_{1} \mid P_{\langle\mathbf{X}\rangle} Y_{2}\right) & =\left([\mathbf{X}] \mathbf{A}_{\mathbf{X}}^{-1}[\mathbf{X}]^{T} \mathbf{A} Y_{2}\right)^{T} \mathbf{A} Y_{1} \\
& =Y_{2}^{T} \mathbf{A}\left([\mathbf{X}] \mathbf{A}_{\mathbf{X}}^{-1}[\mathbf{X}]^{T}\right) \mathbf{A} Y_{1} \\
& =Y_{2}^{T} \mathbf{A} P_{\langle\mathbf{X}\rangle} Y_{1}=\left(P_{\langle\mathbf{X}\rangle} Y_{1} \mid Y_{2}\right),
\end{aligned}
$$

the second statement follows from the first, since $P_{\langle\mathbf{X}\rangle}^{2}=P_{\langle\mathbf{X}\rangle}$. 
Proposition 3.5. Let $\mathbf{X}=\left(X_{1}, \ldots, X_{k}\right), \mathbf{Y}=\left(Y_{1}, \ldots, Y_{m}\right)$ and $\mathbf{Z}=$ $\left(X_{1}, \ldots, X_{k}, Y_{1}, \ldots, Y_{m}\right)$ be linearly independent vectors and $\Delta(\mathbf{X}) \neq 0$. Then

$$
\Delta(\mathbf{Z})=\Delta(\mathbf{X}) \Delta\left(P_{\langle\mathbf{X}\rangle} \mathbf{Y}\right) .
$$

Proof. The determinant of a block matrix is

$$
\operatorname{det}\left[\begin{array}{ll}
\mathbf{A} & \mathbf{C} \\
\mathbf{D} & \mathbf{B}
\end{array}\right]=\operatorname{det} \mathbf{A} \operatorname{det}\left(\mathbf{B}-\mathbf{D A}^{-1} \mathbf{C}\right),
$$

and so

$$
\begin{aligned}
& \Delta(\mathbf{Z})=\operatorname{det}\left[\begin{array}{ll}
{[\mathbf{X}]^{T} \mathbf{A}[\mathbf{X}]} & {[\mathbf{X}]^{T} \mathbf{A}[\mathbf{Y}]} \\
{[\mathbf{Y}]^{T} \mathbf{A}[\mathbf{X}]} & {[\mathbf{Y}]^{T} \mathbf{A}[\mathbf{Y}]}
\end{array}\right] \\
& \left.=\operatorname{det}\left([\mathbf{X}]^{T} \mathbf{A}[\mathbf{X}]\right)\right) \cdot \operatorname{det}\left([\mathbf{Y}]^{T} \mathbf{A}[\mathbf{Y}]\right. \\
& \left.-[\mathbf{Y}]^{T} \mathbf{A}[\mathbf{X}]\left([\mathbf{X}]^{T} \mathbf{A}[\mathbf{X}]\right)^{-1}[\mathbf{X}]^{T} \mathbf{A}[\mathbf{Y}]\right) \\
& =\Delta(\mathbf{X}) \cdot \operatorname{det}\left([\mathbf{Y}]^{T} \mathbf{A}\left([\mathbf{Y}]-P_{\langle\mathbf{X}\rangle}[\mathbf{Y}]\right)\right) \\
& =\Delta(\mathbf{X}) \cdot \operatorname{det}\left([\mathbf{Y}]^{T} \mathbf{A} P_{\langle\mathbf{X}\rangle \perp}[\mathbf{Y}]\right) \\
& =\Delta(\mathbf{X}) \cdot \operatorname{det}\left(\left[P_{\mathbf{X}^{\perp}}(\mathbf{Y})\right]^{T} \mathbf{A}\left[P_{\langle\mathbf{X}\rangle}(\mathbf{Y})\right]\right) \\
& =\Delta(\mathbf{X}) \Delta\left(P_{\langle\mathbf{X}\rangle}(\mathbf{Y})\right) \text {. }
\end{aligned}
$$

The Lie reflection with respect to the subspace $\langle\mathbf{X}\rangle$ is given by

$$
L_{\langle\mathbf{X}\rangle} Y=Y-2 P_{\langle\mathbf{X}\rangle} Y=P_{\langle\mathbf{X}\rangle} Y-P_{\langle\mathbf{X}\rangle^{\perp}} Y .
$$

If $\Delta(\mathbf{X}) \neq 0$, the reflection $L_{\langle\mathbf{X}\rangle}$ is an isomorphism and determines the projective map

$$
L_{\langle\mathbf{x}\rangle}: \mathbb{P}^{n+2} \rightarrow \mathbb{P}^{n+2},
$$

which is the identity on $\langle\mathbf{x}\rangle$ and on $\langle\mathbf{x}\rangle^{\perp}$. It has the following obvious properties:

(i) $L_{\langle\mathbf{X}\rangle}=-L_{\langle\mathbf{X}\rangle}$,

(ii) if $Y \in\langle\mathbf{X}\rangle$, then $L_{\langle\mathbf{X}\rangle} Y=Y$ and if $Y \in\langle\mathbf{X}\rangle^{\perp}$, then $L_{\langle\mathbf{X}\rangle} Y=-Y$,

(iii) $\left(L_{\langle\mathbf{X}\rangle} Y \mid L_{\langle\mathbf{X}\rangle} Z\right)=(Y \mid Z)$,

(iv) if $S \in\langle\mathbf{X}\rangle$, then

$$
(S \mid Y)=-\left(L_{\langle\mathbf{X}\rangle} S \mid Y\right)=-\left(S \mid L_{\langle\mathbf{X}\rangle} Y\right),
$$


(v) if $S \in\langle\mathbf{X}\rangle$, then

$$
(S \mid Y)=\left(L_{\langle\mathbf{X}\rangle} S \mid Y\right)=\left(S \mid L_{\langle\mathbf{X}\rangle} Y\right),
$$

and the reflection $L_{\langle\mathbf{X}\rangle}$ preserves the chart $\mathcal{U}_{s}$ and local coordinates in it, since

$$
\begin{aligned}
L_{\langle\mathbf{X}\rangle} \varphi_{S}(y) & =L_{\langle\mathbf{X}\rangle}\left(\frac{1}{(S \mid Y)} Y\right)=\frac{1}{(Y \mid S)} L_{\langle\mathbf{X}\rangle} Y \\
& =\frac{1}{\left(L_{\langle\mathbf{X}\rangle} Y \mid S\right)} L_{\langle\mathbf{X}\rangle} Y=\varphi_{S}\left(L_{\langle\mathbf{x}\rangle} y\right) .
\end{aligned}
$$

Here are some interesting particular cases:

(i) If either $r \in\langle\mathbf{x}\rangle$ or $r \in\langle\mathbf{x}\rangle^{\perp}$, the reflection $L_{\langle\mathbf{x}\rangle}$ preserves angles.

(ii) If either $w \in\langle\mathbf{x}\rangle$ or $w \in\langle\mathbf{x}\rangle^{\perp}$, the reflection $L_{\langle\mathbf{x}\rangle}$ preserves tangential distance.

(iii) If $x \in\langle r\rangle^{\perp} \backslash \Omega$ is a nonproper cycle, then $L_{x}$ represents the geometric inversion across the proper cycle $\langle x, r\rangle \cap \Omega$ (if it exists).

(iv) The reflection $L_{\langle r\rangle}$ reverses orientation of cycles.

(v) If the special cycle $s$ is of the form $S=\rho W+R, \rho \in \mathbb{R}$, then $L_{s}$ reverses orientation of spheres and changes the radius by $1 / 2 \rho$. Since $s \in\langle w\rangle^{\perp}$, it also preserves tangential distances. Points are thus mapped to spheres of radius $1 / 2 \rho$, while spheres of radius $1 / 2 \rho$ are mapped to points. On planes $L_{s}$ represents a parallel shift and reverses orientation.

The reflection $L_{s}$ from the last example above can be used to give a nice proof of what is the Lie product of two spheres in the case $\left(\varphi_{W}(x) \mid \varphi_{W}(y)\right)>0$ (Figure 4):

Corollary 3.6. In the case when $x$ and $y$ represent spheres $c_{x}$ and $c_{y}$ with radii $\rho_{x}$ and $\rho_{y}$ with no common tangent plane, the product $\left(\varphi_{W}(x) \mid \varphi_{W}(y)\right)$ is $d^{2} / 2$, where $d$ is the half chord of a sphere concentric to $c_{y}$ with radius $\rho_{x}+\rho_{y}$ through the center of $c_{y}$.

Proof. The reflection $L_{s}$, where $S=1 / \rho_{x} W+R$, preserves the chart $\mathcal{U}_{w}$ and local coordinates in it, so

$$
\left(\varphi_{W}(x) \mid \varphi_{W}(y)\right)=\left(\varphi_{W}\left(L_{s} x\right) \mid \varphi_{W}\left(L_{s} y\right)\right)=\frac{d^{2}}{2},
$$


where $L_{s}(x)$ is a point cycle representing the center of $c_{x}$, and $z=L_{s}(y)$ represents the concentric sphere $c_{z}$ to $c_{y}$ with radius $\rho_{x}+\rho_{y}$, so $d$ is the half chord of $c_{z}$ through the center of $c_{x}$.

3.4. Hyperbolic $s$-families. Let $\langle\mathbf{x}, s\rangle \cap \Omega$ be an $s$-family given by linearly independent vectors $\mathbf{X}=\left(X_{1}, \ldots, X_{k}\right), 2 \leq k \leq n$ and $S$. Depending on the sign of determinant $\Delta(\mathbf{X}, S)$ we distinguish three types of $s$-families $\langle\mathbf{x}, s\rangle \cap \Omega$.

(i) If $\Delta(\mathbf{X}, S)>0$, the family is elliptic,

(ii) if $\Delta(\mathbf{X}, S)<0$ the family is hyperbolic and

(iii) if $\Delta(\mathbf{X}, S)=0$ the family is parabolic.

We will be interested only in hyperbolic families since, according to Theorem 3.2, a hyperbolic family as well as its cofamily are both nonempty and thus determine geometric objects.

Definition 3.7. Let $x$ be a nonproper cycle. An intersection of the projective line $\langle x, s\rangle$ with $\Omega$ will be called a projection of $x$ onto $\Omega$ along $s$.

Lemma 3.8. If $(S \mid S) \neq 0$, then a nonproper cycle $x$ has two different projections onto $\Omega$ along $s$ if $\Delta(X, S)<0$, one projection if $\Delta(X, S)=0$ and no projections if $\Delta(X, S)>0$. If $(S \mid S)=0$, then one of the projections is equal to $s$, and a second one exists if and only if $\Delta(X, S) \neq 0$.

Proof. If $(S \mid S) \neq 0$, then the equation

$$
(X+\lambda S \mid X+\lambda S)=(X \mid X)+2 \lambda(X \mid S)+\lambda^{2}(S \mid S)=0
$$

is quadratic with discriminant $-\Delta(X, S)$, so the claim follows. If $(S \mid S)=0$, then $s$ is automatically the projection of an arbitrary $x$ onto $\Omega$ along $s$. A second projection exists if $(X \mid S) \neq 0$; it is the solution of equation (3.4) which is linear in this case. If $(X \mid S)=0$, equation (3.4) has no solutions.

Theorem 3.9. Let $\langle\mathbf{x}, s\rangle \cap \Omega$ be a hyperbolic s-family.

(i) If $(S \mid S)<0$, then each nonproper cycle $x \in\langle\mathbf{x}, s\rangle$ different from $s$ has two projections onto $\Omega$ along s. Each cycle $x \in\langle\mathbf{x}, s\rangle \cap\langle s\rangle^{\perp}$ 
is nonproper with $(X \mid X)>0$. The subspace $\langle\mathbf{X}, S\rangle$ is a direct sum

$$
\langle\mathbf{X}, S\rangle=\langle\mathbf{X}, S\rangle \cap\langle S\rangle^{\perp} \oplus\langle S\rangle .
$$

(ii) If $(S \mid S)=0$, then a cycle $x \in\langle\mathbf{x}, s\rangle \cap\langle s\rangle^{\perp}$ different from $s$ has no projection onto $\Omega$ along $s$ different from $s$, and is nonproper with $(X \mid X)>0$. The subspace $\langle\mathbf{X}, S\rangle$ is a direct sum

$$
\langle\mathbf{X}, S\rangle=\langle\mathbf{X}, S\rangle \cap\langle S\rangle^{\perp} \oplus\langle Y\rangle,
$$

where $y$ is any proper cycle in $\langle\mathbf{x}, s\rangle$ not in $\langle s\rangle^{\perp}$.

\section{Proof.}

(i) Let $(S \mid S)<0$. The restriction of the Lie form to $\langle\mathbf{x}, s\rangle$ has index 1. For any $x \in\langle\mathbf{x}, s\rangle$ where $x$ is nonproper and different from $s$, the same is true also for the subspace $\langle x, s\rangle$, so $\Delta(X, S)<0$, and by the lemma, $x$ has two projections onto $\Omega$ along $s$. If $x \in\langle\mathbf{x}, s\rangle \cap\langle s\rangle^{\perp}$, the projection of $x$ onto $\Omega$ along $s$ has homogeneous coordinates $X+\lambda S$ satisfying the equation

$$
(X+\lambda S \mid X+\lambda S)=(X \mid X)+\lambda^{2}(S \mid S),
$$

so $(X \mid X)=-\lambda^{2}(S \mid S)>0$ and $x$ is nonproper. Clearly, equation (3.5) holds in this case.

(ii) Let $(S \mid S)=0$. In this case, $s \in\langle\mathbf{x}, s\rangle \cap\langle s\rangle^{\perp}$. For each cycle $x \in\langle\mathbf{x}, s\rangle \cap\langle s\rangle^{\perp}$,

$$
\Delta(X, S)=(X \mid X)(S \mid S)-(X \mid S)^{2}=0
$$

so, by the lemma, there is no projection of $x$ onto $\Omega$ along $s$ different from $s$. Let $y \in\langle\mathbf{x}, s\rangle$ be any fixed proper cycles different from $s$. For any $y^{\prime} \neq y, s$, the difference $X=\varphi_{S}(y)-\varphi_{S}\left(y^{\prime}\right)$ is in $\langle S\rangle^{\perp}$, so $Y^{\prime}=X+Y$ and equation (3.6) is valid. Finally, for any $x \in\langle\mathbf{x}, s\rangle \cap\langle s\rangle^{\perp}$ different from $s$, let $y^{\prime}=L_{x} y$, and let $X=\varphi_{S}(y)-\varphi_{S}\left(y^{\prime}\right)$. Then

$$
\begin{aligned}
(X \mid X) & =\left(\varphi_{S}(y)-\varphi_{S}\left(y^{\prime}\right) \mid \varphi_{S}(y)-\varphi_{S}\left(y^{\prime}\right)\right) \\
& =-2\left(\varphi_{S}(y) \mid \varphi_{S}\left(y^{\prime}\right)\right)>0 .
\end{aligned}
$$

3.5. Determinants and geometry. Recall the following well-known determinant from geometry in $\mathbb{R}^{n}$. Let $a_{0}, a_{1}, \ldots, a_{k}$ be the vertices of 
a $k$-dimensional simplex in $\mathbb{R}^{n}$. Then the volume of the simplex is given by the Cayley-Menger determinant

$$
\operatorname{vol}\left(a_{0}, \ldots, a_{k}\right)^{2}=\frac{(-1)^{k-1}}{2^{k} k !^{2}}\left|\begin{array}{cccccc}
0 & d_{01}^{2} & d_{02}^{2} & \cdots & d_{0 k}^{2} & 1 \\
d_{10}^{2} & 0 & d_{12}^{2} & \cdots & d_{1 k}^{2} & 1 \\
\vdots & \vdots & \vdots & \ddots & \vdots & \vdots \\
d_{k 0}^{2} & d_{k 1}^{2} & d_{k 2}^{2} & \cdots & 0 & 1 \\
1 & 1 & 1 & 1 & 1 & 0
\end{array}\right|
$$

where $d_{i j}=\left|a_{i}-a_{j}\right|$. The polar sine of the angle at the vertex $a_{0}$ of the simplex $\left(a_{0}, a_{1}, \ldots, a_{k}\right)$ is defined as:

$$
\operatorname{psin}_{a_{0}}\left(a_{0}, a_{1}, \ldots, a_{k}\right)=k ! \frac{\operatorname{vol}\left(a_{0}, a_{1}, \ldots, a_{k}\right)}{\left|a_{1}-a_{0}\right| \ldots\left|a_{k}-a_{0}\right|} .
$$

It is the ratio of the volume of the given simplex by the volume of the cube with edges from the vertex $a_{0}$ of the same lengths.

Determinants of $r$-families and $w$-families computed in different charts have several interesting geometric interpretations. Following are some examples:

(i) Let $\mathbf{x}=\left(x_{1}, \ldots, x_{k}\right)$ be independent proper cycles, where $2 \leq k \leq$ $n-1$ and $X_{i}=\varphi_{W}\left(x_{i}\right)$ are the homogeneous coordinates in the chart $\mathcal{U}_{w}$.

(ii) The determinant $\Delta(\mathbf{X}, W)$ equals

$$
\Delta(\mathbf{X}, W)=\left|\begin{array}{cccccc}
0 & -\frac{d_{12}^{2}}{2} & -\frac{d_{13}^{2}}{2} & \cdots & -\frac{d_{1 k}^{2}}{2} & 1 \\
-\frac{d_{21}^{2}}{2} & 0 & -\frac{d_{23}^{2}}{2} & \cdots & -\frac{d_{2 k}^{2}}{2} & 1 \\
\vdots & \vdots & \vdots & \ddots & \vdots & \vdots \\
-\frac{d_{k 1}^{2}}{2} & -\frac{d_{k 2}^{2}}{2} & -\frac{d_{k 3}^{2}}{2} & \cdots & 0 & 1 \\
1 & 1 & 1 & 1 & 1 & 0
\end{array}\right|,
$$

where $d_{i j}$ is the tangential distance between the cycle $x_{i}$ and $x_{j}$. If $\Delta(\mathbf{X}, W)<0$, then by $(3.7)$,

$$
\Delta(\mathbf{X}, W)=-(k-1) !^{2} \operatorname{vol}\left(q_{1}, \ldots, q_{k}\right)^{2},
$$

where $q_{1}, \ldots, q_{k}$ are the vertices of the contact simplex, that is, the points of tangency of the cycles of $\mathbf{x}$ to their common 

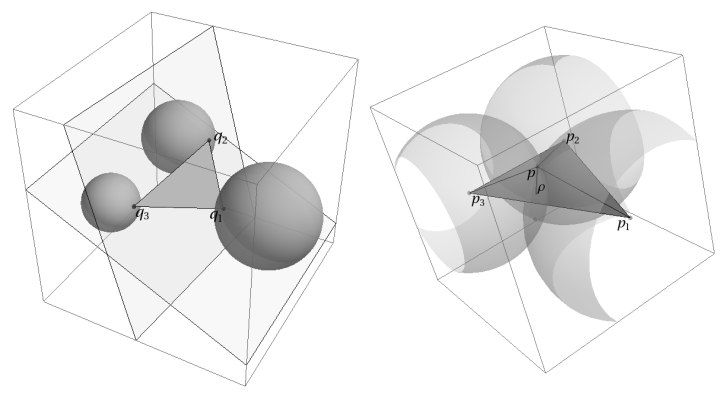

Figure 6 . The contact simplex (left) and the simplex with vertices in the centers of the cycles (right).

tangent plane. The sides of this simplex have lengths equal to the tangential distances between the cycles of $\mathbf{x}$ (Figure 6, left).

(iii) The determinant $\Delta(\mathbf{X}, R, W)$ equals by Proposition 3.5

$$
\Delta(\mathbf{X}, R, W)=(R \mid R) \Delta\left(P_{\langle R\rangle \perp} \mathbf{X}, W\right)=-\Delta\left(P_{\langle R\rangle \perp} \mathbf{X}, W\right),
$$

where $P_{\langle R\rangle} \perp \mathbf{X}$ is formed by the coordinate vectors in the $W$-chart of the point cycles $p_{i}$ corresponding to the centers of $x_{i}$, so

$$
\Delta(\mathbf{X}, R, W)=(k-1) !^{2} \operatorname{vol}\left(p_{1}, \ldots, p_{k}\right)^{2},
$$

gives the squared volume of the simplex with vertices in the centers $p_{i}$ of the cycles $x_{i}$ (Figure 6 , right).

(iv) Finally, let $\left(x_{1}, \ldots, x_{k}\right)$ be a $k$-tuple of intersecting proper cycles, $p$ a point in the common intersection, and $P$ its coordinate vector in the $W$-chart. Then

$$
\begin{aligned}
& \Delta(\mathbf{X}, P, R, W) \\
& =\left|\begin{array}{cccccc}
0 & \left(X_{1} \mid X_{2}\right) & \ldots & 0 & \left(X_{1} \mid R\right) & 1 \\
\left(X_{2} \mid X_{1}\right) & 0 & \cdots & 0 & \left(X_{2} \mid R\right) & 1 \\
\vdots & \vdots & \ddots & \vdots & \vdots & \vdots \\
0 & 0 & 0 & \ldots & 0 & 1 \\
\left(R \mid X_{1}\right) & \left(R \mid X_{2}\right) & \cdots & 0 & -1 & 0 \\
1 & 1 & \cdots & 1 & 0 & 0
\end{array}\right| \\
& =\Delta(\mathbf{X}, R)
\end{aligned}
$$


and

$$
\begin{aligned}
\Delta(\mathbf{X}, P, R, W) & \left.=(R \mid R) \Delta\left(P_{\langle r\rangle}(\mathbf{X}, P)\right), W\right) \\
& =-\Delta\left(P_{\langle r\rangle} \perp \mathbf{X}, P, W\right),
\end{aligned}
$$

where $P_{\langle r\rangle} \perp \mathbf{X}$ represents the point cycles $\left(p_{1}, \ldots, p_{k}\right)$ of the centers of $x_{i}$ expressed in the $W$-chart. By (3.11),

$$
\Delta(\mathbf{X}, P, R, W)=\Delta(\mathbf{X}, R)=-k !^{2} \operatorname{vol}\left(p_{1}, \ldots, p_{k}, p\right)^{2},
$$

where $\operatorname{vol}\left(p_{1}, \ldots, p_{k}, p\right)$ is the volume of the simplex with vertices in the centers $p_{i}$ of the cycles $x_{i}$ and a point $p$ in the intersection.

(v) Let $\mathbf{x}=\left(x_{1}, \ldots, x_{k}\right), 2 \leq k \leq n-1$, be independent proper cycles representing spheres, and let $X_{i}=\varphi_{R}\left(x_{i}\right)$ be homogeneous coordinates in the chart $\mathcal{U}_{r}$. Then $X_{i}=\varphi_{R}\left(x_{i}\right)=\varphi_{W}\left(x_{i}\right) / \rho_{i}$ where $\rho_{i}$ is the radius of the sphere $x_{i}$ and, by (3.8) and (3.13),

$$
\begin{aligned}
\Delta(\mathbf{X}, R) & =-\frac{\Delta\left(\varphi_{W}(\mathbf{x}), R\right)}{\rho_{1}^{2} \ldots \rho_{k}^{2}}=-\frac{k !^{2} \operatorname{vol}\left(p, p_{1}, \ldots, p_{k}\right)^{2}}{\rho_{1}^{2} \cdots \rho_{k}^{2}} \\
& =-\operatorname{psin}_{p}\left(p, p_{1}, \ldots, p_{k}\right)^{2} .
\end{aligned}
$$

On the other hand,

$$
\begin{aligned}
& \left(P_{\langle R\rangle \perp} X_{i} \mid P_{\langle R\rangle \perp} X_{j}\right)=\left(X_{i} \mid X_{j}\right)+1=-\cos \psi_{i j} \\
& \Delta(\mathbf{X}, R)=(R \mid R) \Delta\left(P_{\langle R\rangle \perp} \mathbf{X}\right)=-\Delta\left(P_{\langle R\rangle \perp} \mathbf{X}\right),
\end{aligned}
$$

and it follows that

$$
\begin{aligned}
\Delta(\mathbf{X}, R) & =-\operatorname{psin}\left(p, p_{1}, \ldots, p_{k}\right)^{2} \\
& =-\left|\begin{array}{cccc}
1 & -\cos \psi_{12} & \cdots & -\cos \psi_{1 k} \\
-\cos \psi_{21} & 1 & \cdots & -\cos \psi_{2, k} \\
\vdots & \vdots & \ddots & \vdots \\
-\cos \psi_{k 1} & -\cos \psi_{k 2} & \cdots & 1
\end{array}\right|,
\end{aligned}
$$

where $\psi_{i j}$ is the intersection angle of the spheres $x_{i}$ and $x_{j}$.

3.6. Discriminants. Since the value of the determinant of a family depends on the choice of coordinate vectors, it cannot, by itself, reflect geometric properties of the underlying geometric objects like the radius, center and orientation of a circle given by a Steiner pencil or the vertex, axis and angle at the vertex of a cone given by a cone pencil. These 
are, however, reflected by quotients of determinants. For example, the square of the radius of a sphere given by $x$ is equal to

$$
\rho^{2}=-\frac{\Delta(X, R)}{\Delta(X, R, W)} .
$$

Definition 3.10. Let $S^{\prime}$ be such that $\left(\mathbf{X}, S, S^{\prime}\right)$ are linearly independent, $\Delta(\mathbf{X}, S) \neq 0$ and $\left(S^{\prime} \mid S\right)=0$. The $S^{\prime}$-discriminant of $\langle\mathbf{x}, s\rangle$ is given by

$$
\delta_{S^{\prime}}(\mathbf{x}, s)=\frac{\Delta\left(\mathbf{X}, S, S^{\prime}\right)}{\Delta(\mathbf{X}, S)}
$$

Note that the value of $\delta_{S^{\prime}}(\mathbf{x}, s)$ depends not only on the cycle $s^{\prime}$ but also on the choice of the vector $S^{\prime}$.

For example, if $\mathbf{x}=x$ is a single cycle, the $S^{\prime}$-discriminant of $(x, s)$ is

$$
\begin{aligned}
\delta_{S^{\prime}}(x, s) & =\frac{\Delta\left(X, S, S^{\prime}\right)}{\Delta(X, S)} \\
& =-\frac{1}{(X \mid X)(S \mid S)-(X \mid S)^{2}}\left|\begin{array}{ccc}
(X \mid X) & (X \mid S) & \left(X \mid S^{\prime}\right) \\
(S \mid X) & (S \mid S) & 0 \\
\left(S^{\prime} \mid X\right) & 0 & \left(S^{\prime} \mid S^{\prime}\right)
\end{array}\right| \\
& =\left(S^{\prime} \mid S^{\prime}\right)-\frac{(S \mid S)\left(X \mid S^{\prime}\right)^{2}}{(S \mid S)(X \mid X)-(X \mid S)^{2}}
\end{aligned}
$$

For a fixed subspace $\langle\mathbf{x}, s\rangle$, let $C=P_{\langle\mathbf{x}, S\rangle} S^{\prime}$ denote the Lie projection of the vector $S^{\prime}$ onto the subspace $\langle\mathbf{X}, S\rangle$, and $\mathbf{L}=P_{\langle\mathbf{x}, s\rangle}\langle C\rangle^{\perp}$ the Lie projection of the orthogonal subspace to $\langle C\rangle$ onto $\langle\mathbf{X}, S\rangle$.

Proposition 3.11. If $\Delta(\mathbf{X}, S)<0$,

(i) $\delta_{S^{\prime}}(\mathbf{x}, s)=\left(S^{\prime}-C \mid S^{\prime}-C\right)=\left(S^{\prime} \mid S^{\prime}\right)-(C \mid C)$,

(ii) $c \in\langle s\rangle^{\perp}$ and $\mathbf{l} \subset\left\langle s^{\prime}\right\rangle^{\perp}$,

(iii) $\delta_{S^{\prime}}(\ell, s)=\left(S^{\prime} \mid S^{\prime}\right)$ for any $\ell \in \mathbf{l}$. 
Proof.

(i) By Proposition 3.5,

$$
\begin{aligned}
\delta_{S^{\prime}}(\mathbf{x}, s) & =\frac{\Delta\left(\mathbf{X}, S, S^{\prime}\right)}{\Delta(\mathbf{X}, S)}=\Delta\left(P_{\langle\mathbf{X}, S\rangle \perp} S^{\prime}\right) \\
& =\Delta\left(S^{\prime}-P_{\langle\mathbf{X}, S\rangle}\left(S^{\prime}\right)\right)=\left(S^{\prime}-C \mid S^{\prime}-C\right) \\
& =\left(S^{\prime} \mid S^{\prime}\right)-(C \mid C) .
\end{aligned}
$$

The last equality follows from Proposition 3.4, since $(C \mid C)=$ $\left(C \mid S^{\prime}\right)$.

(ii) $(C \mid S)=\left(P_{\langle\mathbf{X}, S\rangle} S^{\prime} \mid S\right)=\left(S^{\prime} \mid P_{\langle\mathbf{X}, S\rangle} S\right)=\left(S^{\prime} \mid S\right)=0$. For any $L=P_{\langle\mathbf{X}, S\rangle} Y$ where $Y \in\langle C\rangle^{\perp},\left(L \mid S^{\prime}\right)=\left(P_{\langle\mathbf{X}, S\rangle} Y \mid S^{\prime}\right)=(Y \mid$ $\left.P_{\langle\mathbf{X}, S\rangle} S^{\prime}\right)=\left(Y^{\prime} \mid C\right)=0$.

(iii) By the definition, $\Delta\left(L, S, S^{\prime}\right)=\left(S^{\prime} \mid S^{\prime}\right) \Delta(L, S)$.

Theorem 3.12. Let $\langle\mathbf{x}, s\rangle \cap \Omega$ be a hyperbolic s-family and $(S \mid S)<0$. The function

$$
h(x):=\delta_{S^{\prime}}(x, s)
$$

is defined on all $\langle\mathbf{x}, s\rangle \cap \Omega$ and achieves its extreme values on the compact set $\langle\mathbf{x}, s\rangle$ at $c$ and on the subspace $\mathbf{l}$.

Proof. The function $h$ is constant on projective lines through $s$, so $h(x)=h(y)$ for any $x \in\langle\mathbf{x}, s\rangle \cap \Omega$ and $y \in\langle x, s\rangle$. According to Theorem 3.9,

$$
\langle\mathbf{x}, s\rangle=\langle\mathbf{x}, s\rangle \cap\langle s\rangle^{\perp} \oplus\langle s\rangle,
$$

so it suffices to consider values $h(x)$ for $x \in\langle s\rangle^{\perp}$. Then $(X \mid X) \neq 0$ and

$$
h(x)=\left(S^{\prime} \mid S^{\prime}\right)-\frac{\left(X \mid S^{\prime}\right)^{2}}{(X \mid X)}
$$

is well defined. The differential

$$
\begin{aligned}
d h & =-\frac{2\left(X \mid S^{\prime}\right)\left(d X \mid S^{\prime}\right)(X \mid X)-2(d X \mid X)\left(X \mid S^{\prime}\right)^{2}}{(X \mid X)^{2}} \\
& =-2(X \mid S) \frac{\left(d X \mid(X \mid X) S^{\prime}-\left(X \mid S^{\prime}\right) X\right)}{(X \mid X)^{2}}
\end{aligned}
$$


is equal to 0 in two cases: if $\left(X \mid S^{\prime}\right)=0$ so $x \in 1$, and $h(x)=\left(S^{\prime} \mid S^{\prime}\right)$ and if

$$
\left(d X \mid(X \mid X) S^{\prime}-\left(X \mid S^{\prime}\right) X\right)=0
$$

for all $d X \in\langle X, S\rangle$. In this case, $d X=P_{\langle X, S\rangle} d X$,

$$
\begin{aligned}
&\left(P_{\langle\mathbf{X}, S\rangle} d X \mid(X \mid X) S^{\prime}-\left(X \mid S^{\prime}\right) X\right) \\
&=\left(d X \mid(X \mid X) P_{\langle\mathbf{X}, S\rangle} S^{\prime}-\left(X \mid P_{\langle\mathbf{X}, S\rangle} S^{\prime}\right) Y\right) \\
&=(d X \mid(X \mid X) C-(X \mid C) X)=0
\end{aligned}
$$

which implies that $X$ is a multiple of $C$.

3.7. Subcycles and cones. Let us take a closer look at the two cases where $\left(s, S^{\prime}\right)$ is equal to $(r, W)$ and $(w, R)$.

First, let $\langle\mathbf{x}, r\rangle \cap \Omega$ be a hyperbolic $r$-family, that is, a subcycle, and let $S^{\prime}=W$. Let $x$ be a cycle from the family. Then

$$
h(x)=\delta_{W}(x, r)=\frac{\Delta(X, R, W)}{\Delta(X, R)}=-\frac{1}{\rho^{2}},
$$

where $\rho$ is the radius of the corresponding subcycle. Its $W$-discriminant equals

$$
\delta_{W}(\mathbf{x}, r)=-(C \mid C), \quad C=P_{\langle\mathbf{X}, R\rangle} W .
$$

If $c$ is a nonproper cycle and $\delta_{W}(\mathbf{x}, r) \neq 0$, it can be projected onto $\Omega$ along $r$. We will give a geometric meaning to the discriminant $\delta_{W}(\mathbf{x}, r)$, the cycle $c$ and the subspace $\mathbf{l}$.

Assume first that $\delta_{W}(\mathbf{x}, r) \neq 0$. The cycle $c=P_{\langle\mathbf{x}, r\rangle}(w)$ is a nonproper cycle and, according to Proposition 3.11, belongs to the subspace $\langle r\rangle^{\perp}$. According to Theorem 3.9, it can be projected onto $\Omega$ along $r$, and the projection consists of two cycles $c_{1,2}=\langle c, s\rangle \cap \Omega$ which represent one nonoriented sphere with both orientations. By Proposition 3.11,

$$
\delta_{W}(\mathbf{x}, r)=-\frac{(C \mid W)^{2}}{(C \mid C)}=\frac{\Delta\left(C_{i}, R, W\right)}{\Delta\left(C_{i}, R\right)}=-\frac{1}{\rho^{2}},
$$

where $\rho$ is the radius of the cycles $c_{1,2}$ (see Figure 7 on the bottom). By Theorem 3.12, the spheres $c_{1,2}$ have the smallest absolute radius in the family. Also, cycles $\ell \in \mathbf{l}$ can be projected onto the Lie quadric along $r$. Since $(L \mid W)=0$ holds for all $L \in \mathbf{L}$, the projected cycles represent the planes of the family. The family has no point cycles, $(\langle\mathbf{x}, r\rangle \cap \Omega) \cap\langle r\rangle^{\perp}=(\langle\mathbf{x}, r\rangle \cap \Omega) \cap\left(\langle\mathbf{x}, r\rangle^{\perp} \cap \Omega\right)=\emptyset$. 
If $\delta_{W}(\mathbf{x}, r)=0$, the family consists of planes. In this case, the projection $c$ does not exist.
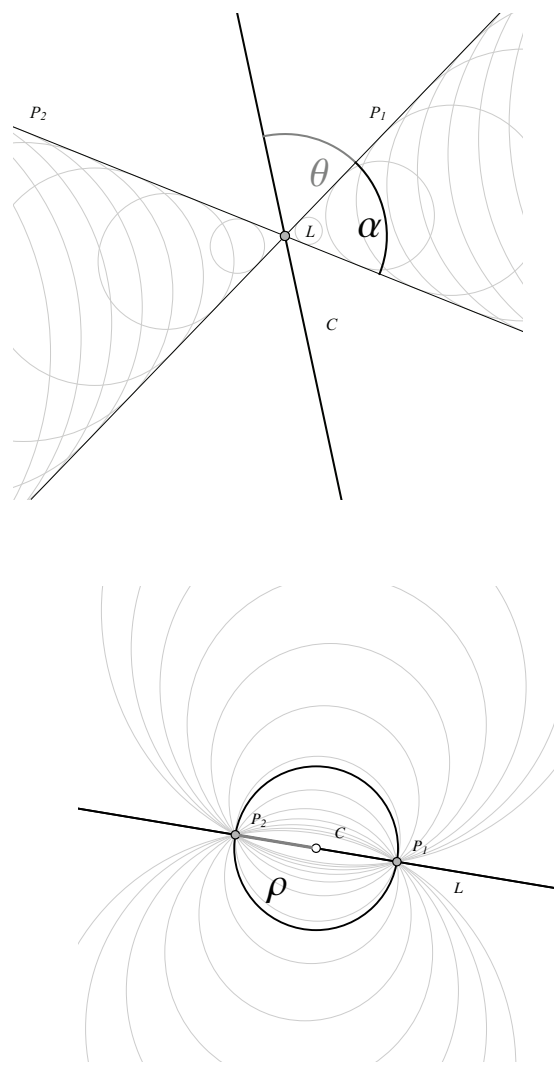

Figure 7. In an $r$-family (top) the cycle $c$ projected onto $\Omega$ along $r$ represents the two oriented spheres with the smallest radius, and the subspace 1 projected onto $\Omega$ along $r$ contains the planes of the family. In a cone family (bottom) $c$ represents the plane orthogonal to the axis of the cone and $\mathbf{l}$ contains the point cycles of the cone, in particular in $\mathbb{R}^{2}$, it is the vertex.

3.7.1. Cones: $s=w$ and $s^{\prime}=r$. The cycles of a hyperbolic $w$-family $\langle\mathbf{x}, w\rangle \cap \Omega$ correspond to a family of geometric cycles with common tangent planes. The points of tangency form a cone in $\mathbb{R}^{n}$, so we will call such a family simply a cone. The $R$-discriminant $\delta_{R}(\mathbf{X}, W)$ of such 
a family is always nonzero since, if $\Delta(\mathbf{X}, W, R)=0$ also $\Delta(\mathbf{X}, W)=0$, which contradicts the hyperbolicity of the family. Using the fact that $(C \mid C)=(R \mid C)$, it is equal to

$$
\begin{aligned}
\delta_{R}(\mathbf{x}, w) & =\left(P_{\langle\mathbf{X}, W\rangle^{\perp}} R \mid P_{\langle\mathbf{X}, W\rangle^{\perp}} R\right) \\
& =(R-C \mid R-C)=-1-(C \mid C) .
\end{aligned}
$$

The cycle $c$ cannot be projected onto $\Omega$ along $w$ since $(C \mid W)=0$. Its projection along $r$ is a nonoriented plane with both orientations, $c_{1,2}$. Using coordinates in the chart $\mathcal{U}_{r}$ we write $C=(\mu, \mathbf{n}, 0,-\rho)$. From $(C \mid C)=(R \mid C)=\rho$, we obtain

$$
\|\mathbf{n}\|^{2}-\rho^{2}=\rho, \quad\|\mathbf{n}\|^{2}=\rho^{2}+\rho .
$$

Let $C_{0}=\left(\mu_{0}, \mathbf{n}_{0}, 0, \rho_{0}\right)=C /\|\mathbf{n}\|$. Then

$$
\rho_{0}= \pm \sqrt{\frac{\rho}{1+\rho}}= \pm \cos \theta
$$

so $\left|\rho_{0}\right| \leq 1$ and $\rho \geq 0$. For an arbitrary cycle $p$ from the cofamily,

$$
\begin{gathered}
P=\varphi_{R}(p)=(\mu, \mathbf{n}, 0,1), \quad\left(C_{0} \mid P\right)=\mathbf{n}_{0} \cdot \mathbf{n}-\rho_{0}=0, \\
\mathbf{n}_{0} \cdot \mathbf{n}= \pm \cos \theta
\end{gathered}
$$

The planes forming the cofamily intersect the two planes $c_{1,2}$ under the angle $\theta$ (see Figure 7), which is complementary to $\alpha / 2$, where $\alpha$ is the vertex angle so

$$
\cos ^{2} \theta=\sin ^{2} \frac{\alpha}{2}=\frac{\rho}{\rho+1} \quad \text { and } \rho=\tan ^{2} \frac{\alpha}{2} .
$$

The discriminant is then

$$
\delta_{R}(\mathbf{x}, w)=-1-(C \mid C)=-1-\rho=-1-\tan ^{2} \frac{\alpha}{2}=-\frac{1}{\cos ^{2} \alpha / 2}
$$

All points of the subspace $\mathbf{l}$ can be projected onto $\Omega$ along $w$ and the projections represent the point cycles (see 3.11). In the case of a cone pencil, the subspace $\mathbf{l}$ has only one cycle representing the vertex of the cone. In the case of a cone family generated by three independent cycles the cofamily has two elements which represent two tangent planes. The proper cycles in $\mathbf{l}$ are points on the line of intersection of these two planes. 
The family has no planes, since

$$
(\langle\mathbf{x}, w\rangle \cap \Omega) \cap\langle w\rangle^{\perp} \subset(\langle\mathbf{x}, w\rangle \cap \Omega) \cap\left(\langle\mathbf{x}, w\rangle^{\perp} \cap \Omega\right)=\emptyset .
$$

4. A family and a cycle. In this section, we focus on the relationship between a cycle and an $s$-family. Throughout this section, we assume that $(S \mid S) \leq 0, \mathbf{x}=\left(x_{1}, \ldots, x_{k}\right), 2 \leq k \leq n-1$, is a list of proper cycles such that $\langle\mathbf{x}, s\rangle \cap \Omega$ is a hyperbolic $s$-family, and $y \notin\langle\mathbf{x}, s\rangle$ is a proper cycle with $(Y \mid S) \neq 0$. We define the discriminant of the triple $(\mathbf{x}, y, s)$ and describe it in the cases $s=r$ and $s=w$ in terms of the special determinants of subsection 3.5. We next consider the discriminant of a triple $\langle x, y, s\rangle$ with $x \in \mathbf{x}$ as a function $h(x)$ and study its critical points.

Definition 4.1. The discriminant of the triple $(\mathbf{x}, y, s)$ is the quotient

$$
\delta(\mathbf{x}, y, s)=\frac{\Delta(\mathbf{X}, Y, S)}{\Delta(\mathbf{X}, S) \Delta(Y, S)} .
$$

Theorem 4.2. The cofamily $\langle\mathbf{x}, s\rangle^{\perp} \cap \Omega$ contains cycles tangent to $y$ if and only if $\delta(\mathbf{x}, y, s) \leq 0$.

Proof. Since $\Delta(\mathbf{X}, S)<0$ and $\Delta(Y, S)=(S \mid S)-(Y \mid S)^{2}<0$, it follows that $\delta(\mathbf{x}, y, s) \leq 0$ precisely when $\Delta(\mathbf{X}, Y, S) \leq 0$. By Theorem 3.2, this is equivalent to $\langle\mathbf{x}, y, s\rangle^{\perp} \cap \Omega \neq \emptyset$, which is true if and only if $\langle\mathbf{x}, s\rangle^{\perp} \cap \Omega$ intersects $\langle y\rangle^{\perp}$. But this means that there exists a $Z \in\langle\mathbf{X}, S\rangle^{\perp}$ such that $(Z \mid Z)=0$ and $(Z \mid Y)=0$, so $z$ is a proper cycle in $\langle\mathbf{x}, s\rangle^{\perp}$ which is tangent to $y$.

Assume that $y \notin\langle s\rangle^{\perp}$. Since $y$ is proper $\Delta(Y, S)=(Y \mid S) \neq 0$, and the discriminant $\delta(x, y, s)$ determines a function

$$
h: \mathbb{P}^{n+1} \backslash\{x \mid \Delta(X, S)=0\} \rightarrow \mathbb{R}, \quad h(x)=\delta(x, y, s) .
$$

The function $h$ is constant on projective lines $\langle x, s\rangle$. If $(S \mid S)<0$, then by Theorem 3.9, any $x \in\langle\mathbf{x}, s\rangle$ has two projections onto $\Omega$ along $s$ and $\Delta(X, S)<0$. So $h(x)$ is defined for all $x \in\langle\mathbf{x}, s\rangle$, and $h$ achieves its maximum and minimum on the compact set $\langle\mathbf{x}, s\rangle \cap \Omega$. If $(S \mid S)=0$, the situation is different since $\Delta(X, S)=0$ for all $x \in\langle s\rangle^{\perp} \cap\langle x, s\rangle \neq \emptyset$, and so $h(x)$ is not bounded on $\langle\mathbf{x}, s\rangle \cap \Omega$. 
Theorem 4.3. The discriminant $\delta(\mathbf{x}, y, s)$ is equal to the value of $h$ at the Lie projection $P_{\langle\mathbf{x}, s\rangle} y$ of $y$ onto $\langle\mathbf{x}, s\rangle$. The cycle $P_{\langle\mathbf{x}, s\rangle} y$ is a critical point of $h$. If $(S \mid S)<0$, then $h(x)$ has a second critical point at a cycle $x_{0} \in\langle\mathbf{x}, s\rangle$, where its value is

$$
h\left(x_{0}\right)=\frac{1}{(S \mid S)} .
$$

Proof. Let $(Y \mid S)=1$, that is, $Y=\varphi_{S}(y)$. Then

$$
h(x)=\frac{(X \mid X)+(-2(X \mid S)+(S \mid S)(X \mid Y))(X \mid Y)}{-(X \mid S)^{2}+(X \mid X)(S \mid S)} .
$$

By Propositions 3.4 and 3.5,

$$
\begin{aligned}
\delta(\mathbf{x}, y, s) & =-\frac{\Delta(\mathbf{X}, Y, S)}{\Delta(\mathbf{X}, S)}=-\Delta\left(P_{\langle\mathbf{X}, S\rangle} Y\right) \\
& =-\Delta\left(Y-P_{\langle\mathbf{X}, S\rangle} Y\right)=\left(P_{\langle\mathbf{X}, S\rangle} Y \mid Y\right) .
\end{aligned}
$$

On the other hand,

$$
\begin{aligned}
\Delta\left(P_{\langle\mathbf{X}, S\rangle} Y, Y, S\right) & =\Delta\left(P_{\langle\mathbf{X}, S\rangle} Y, S\right) \Delta\left(P_{\langle\mathbf{X}, S\rangle} \perp\right) \\
& =-\left(P_{\langle\mathbf{X}, S\rangle} Y \mid Y\right) \Delta\left(P_{\langle\mathbf{X}, S\rangle} Y, S\right),
\end{aligned}
$$

so

$$
h\left(P_{\langle\mathbf{x}, s\rangle}(y)\right)=-\frac{\Delta\left(P_{\langle\mathbf{X}, S\rangle} Y, Y, S\right)}{\Delta\left(P_{\langle\mathbf{X}, S\rangle} Y, S\right)}=\left(P_{\langle\mathbf{X}, S\rangle} Y \mid Y\right)=\delta(\mathbf{x}, s, y),
$$

and the first statement is true.

Now let $x \in\langle\mathbf{x}, s\rangle$ be different from $s$, and let $(X \mid S)=1$. Since $(Y \mid S)=1$, also

$$
\left(P_{\langle X, S\rangle} Y \mid S\right)=\left(Y \mid P_{\langle X, S\rangle} S\right)=(Y \mid S)=1 .
$$

We will consider the cases when $(S \mid S)<0$ and $(S \mid S)=0$ separately.

- In the case $(S \mid S)=0$, the function $h(x)$ is defined for $x \in\langle\mathbf{x}, s\rangle \backslash\langle s\rangle^{\perp}$ and

$$
h(x)=(X \mid X)-2(X \mid Y),
$$

so

$$
d h=2(d X \mid X)-2(d X \mid Y)=2(d X \mid X-Y) .
$$


For $X=P_{\langle\mathbf{X}, S\rangle} Y$,

$$
\begin{aligned}
(d X \mid X-Y) & =\left(d X \mid P_{\langle\mathbf{X}, S\rangle} Y-Y\right) \\
& =-\left(d X \mid P_{\langle\mathbf{X}, S\rangle}{ }^{\perp} Y\right)=0
\end{aligned}
$$

for all $d X \in\langle\mathbf{X}, S\rangle$.

- In the second case, we may assume that $(S \mid S)=-1$. Since $h(x)$ is constant on projective lines and, since every projective line $\langle x, s\rangle$ intersects $\langle s\rangle^{\perp}$, we may pick $x \in\langle\mathbf{x}, s\rangle \cap\langle s\rangle^{\perp}$. Then,

$$
h(x)=-1+\frac{(X \mid Y)^{2}}{(X \mid X)},
$$

and

$d h=-\frac{2(X \mid Y)}{(X \mid X)^{2}}((X \mid X)(d X \mid Y)-(X \mid Y)(d X \mid X))$

equals 0 if either $(X \mid Y)=0$ or $(d X \mid(X \mid X) Y-(X \mid Y) X)=$ 0 .

Let $X=P_{\langle\mathbf{X}, S\rangle} Y$. Then

$$
\begin{aligned}
& (d X \mid(X \mid X) Y-(X \mid Y) X) \\
& =\left(P_{\langle\mathbf{X}, S\rangle} Y \mid Y\right)\left(d X \mid Y-P_{\langle\mathbf{X}, S\rangle} Y\right) \\
& =0
\end{aligned}
$$

for all $d X \in\langle\mathbf{X}, S\rangle$, since $Y-P_{\langle\mathbf{X}, S\rangle} Y=P_{\langle\mathbf{X}, S\rangle}{ }^{\perp} Y$, so $X$ is a critical point of $h$.

The second critical point is at the cycle $x_{0}$ where $\left(X_{0} \mid Y\right)=$ 0 and $h\left(x_{0}\right)=-1$.

Let us consider two special cases, $S=R$ and $S=W$.

(i) The case of $S=R$. Without loss of generality, we can assume that all cycles $\mathbf{x}$ represent spheres. Then

$$
\begin{aligned}
\delta(\mathbf{x}, y, r) & =\frac{\Delta\left(\varphi_{R}(\mathbf{x}), \varphi_{R}(y), R\right)}{\Delta\left(\varphi_{R}(\mathbf{x}), R\right) \Delta\left(\varphi_{R}(y), R\right)} \\
& =-\frac{\Delta\left(\varphi_{W}(\mathbf{x}), \varphi_{W}(y), R\right)}{\Delta\left(\varphi_{W}(\mathbf{x}), R\right)\left(\varphi_{W}(y) \mid R\right)^{2}}=
\end{aligned}
$$




$$
\begin{aligned}
& =-\frac{(k+1)^{2} \operatorname{vol}\left(q_{1}, \ldots, q_{k}, q_{y}, p\right)}{\operatorname{vol}\left(q_{1}, \ldots, q_{k}, p\right)^{2} \rho^{2}} \\
& =-\frac{v^{2}}{\rho^{2}}=-\sin ^{2} \alpha,
\end{aligned}
$$

where $q_{i}$ and $q_{y}$ are centers of the spheres $x_{i}, i=1, \ldots, k$, and $y$, respectively, $p$ is a point in the intersection, $\rho$ is the radius of the cycle $y, v$ is the height of the simplex spanned by $\left(q_{1}, \ldots, q_{k}, q_{y}, p\right)$ to the base simplex spanned by $\left(q_{1}, \ldots, q_{k}, p\right)$ and $\alpha$ is the angle between the plane of the base simplex and the line segment which connects the center $q_{y}$ with an intersection point $p$.

(ii) The case of $S=W$. In this case,

$$
\begin{aligned}
\delta(\mathbf{x}, y, w) & =\frac{\Delta\left(\varphi_{W} \mathbf{x}, \varphi_{W}(y), W\right)}{\Delta\left(\varphi_{W}(\mathbf{x}), W\right) \Delta\left(\varphi_{W}(y), W\right)} \\
& =-\frac{k^{2} \operatorname{vol}\left(q_{1}, \ldots, q_{k}, q_{y}\right)^{2}}{\operatorname{vol}\left(q_{1}, \ldots, q_{k}\right)^{2}}=-v^{2},
\end{aligned}
$$

where the points $\left(q_{1}, \ldots, q_{k}, q_{y}\right)$ span the contact simplex that lies in the common tangent plane of the cycles $(\mathbf{x}, y),\left(q_{1}, \ldots, q_{k}\right)$ span the contact simplex of $\mathbf{x}$ (see (3.9)) and $v$ is the height of the first simplex above to the second. The height $v$ is the tangential distance from the cycle $y$ to the cone.

5. Two families. In this section, we consider the relation between two $s$-families of the same dimension. We define the discriminant of the triple $(\mathbf{x}, \mathbf{y}, s)$ and show that it can be expressed in terms of the eigenvalues of the product $P_{\langle\mathbf{X}, S\rangle} P_{\langle\mathbf{Y}, S\rangle}$. We discuss the critical points of the function obtained by considering the determinant of triples $(x, y, s), x \in\langle\mathbf{x}, s\rangle, y \in\langle\mathbf{y}, s\rangle$ as a function of $(x, y)$ and show that they are closely associated to the fixed points of the products $P_{\langle\mathbf{x}, s\rangle} P_{\langle\mathbf{y}, s\rangle}$ and $P_{\langle\mathbf{y}, s\rangle} P_{\langle\mathbf{x}, s\rangle}$ (which correspond to the eigenvectors of the products $P_{\langle\mathbf{X}, S\rangle} P_{\langle\mathbf{Y}, S\rangle}$ and $\left.P_{\langle\mathbf{Y}, S\rangle} P_{\langle\mathbf{X}, S\rangle}\right)$. We also give a geometric interpretation in the two cases $s=r$ and $s=w$.

Definition 5.1. The discriminant of two hyperbolic $s$-families $\langle\mathbf{x}, s\rangle \cap \Omega$ and $\langle\mathbf{y}, s\rangle \cap \Omega$ spanned by linearly independent proper cycles $\mathbf{x}=$ 
$\left(x_{1}, \ldots, x_{k}\right)$ and $\mathbf{y}=\left(y_{1}, \ldots, y_{k}\right)$ is defined by

$$
\delta(\mathbf{x}, \mathbf{y}, s)=\frac{\Delta(\mathbf{X}, \mathbf{Y}, S)}{\Delta(\mathbf{X}, S) \Delta(\mathbf{Y}, S)}
$$

Because of the hyperbolicity of the two families and the linear independence of the spanning cycles, the family $\langle\mathbf{x}, \mathbf{y}, s\rangle \cap \Omega$ is hyperbolic if and only if $\delta(\mathbf{x}, \mathbf{y}, s)<0$.

Proposition 5.2. The value $\delta(\mathbf{x}, \mathbf{y}, s)$ of the discriminant of two hyperbolic $s$-families $\langle\mathbf{x}, s\rangle \cap \Omega$ and $\langle\mathbf{y}, s\rangle \cap \Omega$ is equal to 0 if either contain a common cycle $z \in\langle\mathbf{x}, s\rangle \cap\langle\mathbf{y}, s\rangle$ or the corresponding cofamilies have a nonempty intersection.

Proof. In the first case, the intersection $\langle\mathbf{X}, S\rangle \cap\langle\mathbf{Y}, S\rangle$ contains a vector $Z \notin\langle S\rangle$, so the columns of $\Delta(\mathbf{X}, \mathbf{Y}, S)$ are linearly dependent. In the second case, the equation $(Z \mid Z)=0$ has a nontrivial solution in the subspace $\langle\mathbf{X}, \mathbf{Y}, S\rangle$. The resulting homogeneous system

$$
(Z \mid S)=0, \quad\left(Z \mid X_{i}\right)=0, \quad\left(Z \mid Y_{i}\right)=0, \quad i=1, \ldots, k,
$$

thus has a nontrivial solution, and the coefficient matrix of this system, which equals $\Delta(\mathbf{X}, \mathbf{Y}, S)$, must be 0 .

\subsection{Eigenvalues and eigenvectors of a product of two projec-} tors. We will need some properties of the eigenvectors and eigenvalues of the products $P_{\langle\mathbf{X}, S\rangle} P_{\langle\mathbf{Y}, S\rangle}$ and $P_{\langle\mathbf{Y}, S\rangle} P_{\langle\mathbf{X}, S\rangle}$ which we prove in this section. Since $S \in\langle\mathbf{X}, S\rangle \cap\langle\mathbf{Y}, S\rangle$, one of the eigenvalues is 1 , and one of the corresponding eigenvectors is $S$. The eigenvectors corresponding to the eigenvalue 1 are precisely the vectors in the intersection $\langle\mathbf{X}, S\rangle \cap\langle\mathbf{Y}, S\rangle$.

Proposition 5.3. The products of the Lie projectors $P_{\langle\mathbf{X}, S\rangle} P_{\langle\mathbf{Y}, S\rangle}$ and $P_{\langle\mathbf{Y}, S\rangle} P_{\langle\mathbf{X}, S\rangle}$ have the same eigenvalues. If $E$ is an eigenvector of $P_{\langle\mathbf{X}, S\rangle} P_{\langle\mathbf{Y}, S\rangle}$, then $F=P_{\langle\mathbf{Y}, S\rangle} E$ is an eigenvector of $P_{\langle\mathbf{Y}, S\rangle} P_{\langle\mathbf{X}, S\rangle}$. Eigenvectors which belong to different nonzero eigenvalues are Lie orthogonal. 
Proof. Multiplying both sides of the equation $P_{\langle\mathbf{X}, S\rangle} P_{\langle\mathbf{Y}, S\rangle} E=\lambda E$ by $P_{\langle\mathbf{Y}, S\rangle}$, we get

$$
P_{\langle\mathbf{Y}, S\rangle} P_{\langle\mathbf{X}, S\rangle} P_{\langle\mathbf{Y}, S\rangle} E=\lambda P_{\langle\mathbf{Y}, S\rangle} E .
$$

If $P_{\langle\mathbf{Y}, S\rangle} E=F$, it follows that $P_{\langle\mathbf{Y}, S\rangle} P_{\langle\mathbf{X}, S\rangle} F=\lambda F$. So the vector $F$ is an eigenvector of $P_{\langle\mathbf{Y}, S\rangle} P_{\langle\mathbf{X}, S\rangle}$ corresponding to the same eigenvalue $\lambda$.

Let $E_{i}$ and $E_{j}$ be eigenvectors of $P_{\langle\mathbf{X}, S\rangle} P_{\langle\mathbf{Y}, S\rangle}$, corresponding to eigenvalues $\lambda_{i}$ and $\lambda_{j}$, respectively, where $\lambda_{i} \neq \lambda_{j}$ and $\lambda_{i}, \lambda_{j} \neq 0$. Then

$$
\left(P_{\langle\mathbf{X}, S\rangle} P_{\langle\mathbf{Y}, S\rangle} E_{i} \mid P_{\langle\mathbf{X}, S\rangle} P_{\langle\mathbf{Y}, S\rangle} E_{j}\right)=\lambda_{i} \lambda_{j}\left(E_{i} \mid E_{j}\right) .
$$

On the other hand,

$$
\begin{aligned}
& \left(P_{\langle\mathbf{X}, S\rangle} P_{\langle\mathbf{Y}, S\rangle} E_{i} \mid P_{\langle\mathbf{X}, S\rangle} P_{\langle\mathbf{Y}, S\rangle} E_{j}\right) \\
& =\left(P_{\langle\mathbf{Y}, S\rangle} P_{\langle\mathbf{X}, S\rangle} P_{\langle\mathbf{Y}, S\rangle} E_{i} \mid E_{j}\right) \\
& =\left(P_{\langle\mathbf{Y}, S\rangle} P_{\langle\mathbf{X}, S\rangle} P_{\langle\mathbf{Y}, S\rangle} E_{i} \mid P_{\langle\mathbf{X}, S\rangle} E_{j}\right) \\
& =\left(P_{\langle\mathbf{X}, S\rangle} P_{\langle\mathbf{Y}, S\rangle} P_{\langle\mathbf{X}, S\rangle} P_{\langle\mathbf{Y}, S\rangle} E_{i} \mid E_{j}\right) \\
& =\lambda_{i}^{2}\left(E_{i} \mid E_{j}\right) .
\end{aligned}
$$

Since $\lambda_{i} \neq \lambda_{j}$, it follows that $\left(E_{i} \mid E_{j}\right)=0$.

From now on, let $\mathbf{E}$ and $\mathbf{F}$ denote lists of linearly independent eigenvectors of $P_{\langle\mathbf{X}, S\rangle} P_{\langle\mathbf{Y}, S\rangle}$ and $P_{\langle\mathbf{Y}, S\rangle} P_{\langle\mathbf{X}, S\rangle}$, respectively, which are independent from $S$.

Proposition 5.4. Assume that $\delta(\mathbf{x}, \mathbf{y}, s)<0$.

(i) The eigenvalues of the products $P_{\langle\mathbf{X}, S\rangle} P_{\langle\mathbf{Y}, S\rangle}$ and $P_{\langle\mathbf{Y}, S\rangle} P_{\langle\mathbf{X}, S\rangle}$ different from zero are positive. The restriction of the Lie form to $\langle\mathbf{E}\rangle$ and $\langle\mathbf{F}\rangle$ is positive definite.

(ii) If $E \in \mathbf{E}$ and $F \in \mathbf{F}$ are eigenvectors belonging to the same eigenvalue such that $(E \mid E)=1$ and $(F \mid F)=1$, then $(E \mid F)=\sqrt{\lambda}$ where $\lambda$ is the corresponding eigenvalue.

(iii) If $(S \mid S)<0$, then all eigenvalues are nondegenerate, and the corresponding eigenvectors span the subspaces $\langle\mathbf{X}, S\rangle$ and $\langle\mathbf{Y}, S\rangle$. All cycles $e \in\langle\mathbf{e}\rangle$ and $f \in\langle\mathbf{f}\rangle$ different from $s$ can be projected onto the Lie quadric along $s$. 
If $(S \mid S)=0$ the eigenvalue 1 is degenerate and the cycles, corresponding to the eigenvectors independent from $S$, cannot be projected onto the Lie quadric along s.

\section{Proof.}

(i) Since $\delta(\mathbf{x}, \mathbf{y}, s)<0, S$ is the only eigenvector corresponding to 1 and, by Proposition 5.3, $\langle\mathbf{E}\rangle \subset S^{\perp}$ and also $\langle\mathbf{F}\rangle \subset S^{\perp}$. By Theorem 3.9, this implies that $(E \mid E)>0$ and $(F \mid F)>0$ for all $E \in \mathbf{E}$ and $F \in \mathbf{F}$, and the restriction of the Lie form to the span $\langle\mathbf{E}\rangle$ and $\langle\mathbf{F}\rangle$ of eigenvectors is positive definite. Also,

$$
\begin{aligned}
(F \mid F) & =\left(P_{\langle\mathbf{Y}, S\rangle} E \mid P_{\langle\mathbf{Y}, S\rangle} E\right)=\left(P_{\langle\mathbf{Y}, S\rangle} E \mid E\right) \\
& =\left(P_{\langle\mathbf{Y}, S\rangle} E \mid P_{\langle\mathbf{X}, S\rangle} E\right)=\left(P_{\langle\mathbf{X}, S\rangle} P_{\langle\mathbf{Y}, S\rangle} E \mid E\right) \\
& =\lambda(E \mid E)>0
\end{aligned}
$$

so $\lambda>0$.

(ii) If $E$ and $F$ are eigenvectors belonging to the same eigenvalue $\lambda$ and $(E \mid E)=1$ and $(F \mid F)=1$, then $P_{\langle\mathbf{Y}, S\rangle} E=c F$, $(c F \mid c F)=c^{2}$. On the other hand,

$$
\begin{aligned}
(c F \mid c F) & =\left(P_{\langle\mathbf{Y}, S\rangle} E \mid P_{\langle\mathbf{Y}, S\rangle} E\right)=\left(P_{\langle\mathbf{Y}, S\rangle} E \mid E\right) \\
& =\left(P_{\langle\mathbf{Y}, S\rangle} E \mid P_{\langle\mathbf{X}, S\rangle} E\right)=\left(P_{\langle\mathbf{X}, S\rangle} P_{\langle\mathbf{Y}, S\rangle} E \mid E\right) \\
& =\lambda,
\end{aligned}
$$

so $c=\sqrt{\lambda}$. Also,

$$
\begin{aligned}
(E \mid F) & =\left(E \mid \frac{1}{\sqrt{\lambda}} P_{\langle\mathbf{Y}, S\rangle} E\right) \\
& =\left(P_{\langle\mathbf{X}, S\rangle} E \mid \frac{1}{\sqrt{\lambda}} P_{\langle\mathbf{Y}, S\rangle} E\right) \\
& =\left(E \mid \frac{1}{\sqrt{\lambda}} P_{\langle\mathbf{X}, S\rangle} P_{\langle\mathbf{Y}, S\rangle} E\right) \\
& =\sqrt{\lambda} .
\end{aligned}
$$

(iii) This follows directly from Theorem 3.9.

5.2. The discriminant. Let $\langle\mathbf{x}, s\rangle \cap \Omega$ and $\langle\mathbf{y}, s\rangle \cap \Omega$ be two $k$ dimensional hyperbolic families with $\delta(\mathbf{x}, \mathbf{y}, s)<0$. We discuss the cases $(S \mid S)<0$ and $(S \mid S)=0$ separately. 
5.2.1. The case of $(S \mid S)<0$. In this case $(\mathbf{E}, S)$ and $(\mathbf{F}, S)$ are bases of $\langle\mathbf{X}, S\rangle$ and $\langle\mathbf{Y}, S\rangle$ consisting of eigenvectors of the products $P_{\langle\mathbf{X}, S\rangle} P_{\langle\mathbf{Y}, S\rangle}$ and $P_{\langle\mathbf{Y}, S\rangle} P_{\langle\mathbf{X}, S\rangle}$, respectively, such that $\left(E_{i} \mid E_{j}\right)=\delta_{i j}$, $\left(F_{i} \mid F_{j}\right)=\delta_{i j}, F_{i}=P_{\langle\mathbf{X}, S\rangle} E_{i}$ and $\left(E_{i} \mid F_{j}\right)=\sqrt{\lambda_{i}} \delta_{i j}$ for all $i, j=1, \ldots, k$. Each cycle $e_{i} \in \mathbf{e}$ and $f_{j} \in \mathbf{f}$ can be projected onto the Lie quadric in the direction of $s$. The discriminant equals

$$
\begin{aligned}
\delta(\mathbf{x}, \mathbf{y}, s) & =\left|\begin{array}{cccccc}
1 & 0 & \cdots & \sqrt{\lambda_{1}} & 0 & 0 \\
0 & 1 & \cdots & 0 & \sqrt{\lambda_{2}} & 0 \\
\ldots \ldots & \ldots \ldots \ldots \ldots \ldots \ldots \ldots \ldots \ldots \ldots \\
\sqrt{\lambda_{1}} & 0 & \cdots & 1 & 0 & 0 \\
0 & \sqrt{\lambda_{2}} & \cdots & 0 & 1 & 0 \\
0 & 0 & \cdots & 0 & 0 & -1
\end{array}\right| \\
& =\left|\begin{array}{ccc}
\mathbf{I} & \boldsymbol{\Lambda} & \mathbf{0} \\
\boldsymbol{\Lambda} & \mathbf{I} & \mathbf{0} \\
\mathbf{0}^{T} & \mathbf{0}^{T} & 1
\end{array}\right|=-\left|\mathbf{I}-\boldsymbol{\Lambda}^{2}\right| \\
& =-\prod_{i=1}^{k}\left(1-\lambda_{i}\right),
\end{aligned}
$$

where

$$
\Lambda=\left[\begin{array}{cccc}
\sqrt{\lambda_{1}} & 0 & \cdots & 0 \\
0 & \sqrt{\lambda_{2}} & \cdots & 0 \\
\ldots \ldots & \ldots & \ldots & \ldots \\
0 & 0 & \cdots & \sqrt{\lambda_{k}}
\end{array}\right]
$$

and $\mathbf{I}$ is the identity matrix of the size $k \times k$.

Let $S=R$. Each cycle $e_{i} \in \mathbf{E}$ and $f_{i} \in \mathbf{F}$ belongs to the space $\langle r\rangle^{\perp}$ and can be projected onto the Lie quadric in the direction of the $r$. The projections $\widehat{e}_{i}$ and $\widehat{f}_{i}$ have homogeneous coordinates $\widehat{E}_{i}=E_{i}-R$ and $\widehat{F}_{i}=F_{i}-R\left(\right.$ since $\left(E_{i} \mid E_{i}\right)=1$ and $\left(F_{i} \mid F_{i}=1\right)$ and

$$
\left(\widehat{E}_{i} \mid \widehat{F}_{i}\right)=\left(E_{i}-R \mid F_{i}-R\right)=\sqrt{\lambda_{i}}-1 .
$$

Since $\left(\widehat{E}_{i} \mid R\right)=\left(\widehat{F}_{i} \mid R\right)=1$ it follows that $\widehat{E}_{i}$ and $\widehat{F}_{i}$ are homogeneous coordinates in the chart $\mathcal{U}_{r}$ and, by $(2.4), \sqrt{\lambda_{i}}=-\cos \alpha_{i}$, where $\alpha_{i}$ is the angle of intersection of the geometric cycles corresponding to $\widehat{e}_{i} \in\langle\mathbf{x}, s\rangle \cap \Omega$ and $\widehat{f}_{i} \in\langle\mathbf{y}, s\rangle \cap \Omega$. The eigenvalue $\lambda_{i}$ is thus equal to $\cos ^{2} \alpha_{i}$. 
5.2.2. The case of $(S \mid S)=0$. Since 1 is a degenerate eigenvalue, the eigenvectors $(\mathbf{E}, S)$ and $(\mathbf{F}, S)$ do not span the subspaces $\langle\mathbf{X}, S\rangle$ and $\langle\mathbf{Y}, S\rangle$, so there exist vectors $T$ and $U$ such that $\langle T, S\rangle=\langle\mathbf{E}\rangle^{\perp} \cap\langle\mathbf{X}, \mathbf{S}\rangle$ and $\langle U, S\rangle=\langle\mathbf{F}\rangle^{\perp} \cap\langle\mathbf{Y}, \mathbf{S}\rangle$.

Let $\widehat{t}$ and $\widehat{u}$ be the projections of $t$ and $u$ onto the quadric along $s$, and $\widehat{T}$ and $\widehat{U}$ homogeneous coordinates in the chart $\mathcal{U}_{s}$ so that a basis of the space $\langle T, S\rangle,(\widehat{T}, S)$ and similarly $(\widehat{U}, S)$. Then $\langle\mathbf{E}, \widehat{T}, S\rangle=\langle\mathbf{X}, S\rangle$ and $\langle\mathbf{F}, \widehat{U}, S\rangle=\langle\mathbf{Y}, S\rangle$, and the discriminant is

$$
\delta(\mathbf{x}, \mathbf{y}, s)=\left|\begin{array}{ccc}
\mathbf{I} & \boldsymbol{\Lambda} & \mathbf{0} \\
\mathbf{\Lambda} & \mathbf{I} & \mathbf{0} \\
\mathbf{0}^{T} & \mathbf{0}^{T} & D
\end{array}\right|,
$$

where

$$
\Lambda=\left[\begin{array}{cccc}
\sqrt{\lambda_{1}} & 0 & \cdots & 0 \\
0 & \sqrt{\lambda_{2}} & \cdots & 0 \\
\ldots \ldots & \ldots & \ldots & \ldots \\
0 & 0 & \cdots & \sqrt{\lambda_{k-1}}
\end{array}\right]
$$

I is the identity matrix of size of $k-1 \times k-1$ and

$$
D=\left[\begin{array}{ccc}
0 & (\widehat{T} \mid \widehat{U}) & 1 \\
(\widehat{T} \mid \widehat{U}) & 0 & 1 \\
1 & 1 & 0
\end{array} \mid .\right.
$$

Thus,

$$
\delta(\mathbf{x}, \mathbf{y}, s)=2(\widehat{T} \mid \widehat{U}) \prod_{i=1}^{k-1}\left(1-\lambda_{i}\right)
$$

Let $S=W$. Cycles $e \in \mathbf{e}$ and $f \in \mathbf{f}$ belong to the space $\langle w\rangle^{\perp}$ and cannot be projected onto the Lie quadric along $w$. Let $\widehat{e}_{i}$ and $\widehat{f}_{i}$ be the projections along $r$. These cycles represent the planes of the two $w$-families. Local coordinates in the chart $\mathcal{U}_{r}$ are $\widehat{E}_{i}=E_{i}-R$ and $\widehat{F}_{i}=F_{i}-R$, so $\left(\widehat{E}_{i} \mid \widehat{F}_{i}\right)=1-\lambda_{i}=\sin ^{2} \alpha_{i}$ where $\alpha_{i}$ the angle between the planes $\widehat{e}_{i}$ and $\widehat{f}_{i}$. On the other hand, $\widehat{T}$ and $\widehat{U}$ are local coordinates in $\mathcal{U}_{w},(\widehat{T} \mid \widehat{U})=-d^{2} / 2$, where $d$ is the tangential distance between the cycles $\widehat{t}$ and $\widehat{u}$. As we will see later, this tangential distance is the minimal tangential distance between the cycles of the families $\langle\mathbf{x}, w\rangle \cap \Omega$ 
and $\langle\mathbf{y}, w\rangle \cap \Omega$. Thus,

$$
\delta(\mathbf{x}, \mathbf{y}, w)=-d^{2} \prod_{i=1}^{k-1} \sin ^{2} \alpha_{i}
$$

5.3. Critical points. Let $\langle\mathbf{x}, s\rangle \cap \Omega$ and $\langle\mathbf{y}, s\rangle \cap \Omega$ be $k$-parametric hyperbolic families, such that $\delta(\mathbf{x}, \mathbf{y}, s)<0$. The two products $P_{\langle\mathbf{X}, S\rangle} P_{\langle\mathbf{Y}, S\rangle}$ and $P_{\langle\mathbf{Y}, S\rangle} P_{\langle\mathbf{X}, S\rangle}$ both have rank $k+1$, and there are at most $k+1$ nonzero eigenvalues. One of them is 1 , and its corresponding eigenvector is $S$.

Now consider the function

$$
h(x, y)=\frac{\Delta(X, Y, S)}{\Delta(X, S) \Delta(Y, S)}
$$

where $x \in\langle\mathbf{x}, s\rangle$ and $y \in\langle\mathbf{y}, s\rangle$ vary independently.

Theorem 5.5. The function $h(x, y)$ restricted to $\langle\mathbf{x}, s\rangle \cap \Omega \times\langle\mathbf{y}, s\rangle \cap \Omega$ has at least one critical point. If $(e, f)$ is a critical point of $h(x, y)$, then $e$ is a critical point of

$$
h_{1}(y)=\frac{\Delta(\mathbf{X}, Y, S)}{\Delta(\mathbf{X}, S) \Delta(Y, S)}
$$

and $f$ is a critical point of

$$
h_{2}(x)=\frac{\Delta(X, \mathbf{Y}, S)}{\Delta(X, S) \Delta(\mathbf{Y}, S)} .
$$

(i) If $(S \mid S)<0$, the function $h(x, y)$ restricted to $\langle\mathbf{x}, s\rangle \cap \Omega \times\langle\mathbf{y}, s\rangle \cap$ $\Omega$ has a critical point at a pair $(\widehat{e}, \widehat{f})$ obtained by projecting a fixed point $e$ of $P_{\langle\mathbf{x}, s\rangle} P_{\langle\mathbf{y}, s\rangle}$ and $f$ of $P_{\langle\mathbf{y}, s\rangle}(e)$ of $P_{\langle\mathbf{y}, s\rangle} P_{\langle\mathbf{x}, s\rangle}$ onto $\langle\mathbf{x}, s\rangle \cap \Omega$ and $\langle\mathbf{y}, s\rangle \cap \Omega$, respectively, along s. All critical points are of this type.

(ii) If $(S \mid S)=0$, there exists a unique fixed projective line $\langle t, s\rangle$ of $P_{\langle\mathbf{x}, s\rangle} P_{\langle\mathbf{y}, s\rangle}$ and a unique fixed projective line $\langle u, s\rangle$ of $P_{\langle\mathbf{y}, s\rangle} P_{\langle\mathbf{x}, s\rangle}$. These intersect the Lie quadric in points $\widehat{t}$ and $\widehat{u}$. The point $(\widehat{t}, \widehat{u})$ is the only critical point of $h(x, y)$ restricted to the $\langle\mathbf{x}, s\rangle \cap \Omega \times$ $\langle\mathbf{y}, s\rangle \cap \Omega$. 
Proof. Consider the function

$$
\begin{aligned}
h_{1}(y) & =\frac{\Delta(\mathbf{X}, Y, S)}{\Delta(\mathbf{X}, S) \Delta(Y, S)}=\frac{\Delta\left(P_{\langle\mathbf{X}, \mathbf{S}\rangle} \perp Y\right)}{\Delta(Y, S)} \\
& =\frac{(Y \mid Y)-\left(Y \mid P_{\langle\mathbf{X}, S\rangle} Y\right)}{\Delta(Y, S)} .
\end{aligned}
$$

(i) Let $(S \mid S)<0$, and let $(e, f)$ be such that $e$ is a fixed point of $P_{\langle\mathbf{x}, s\rangle} P_{\langle\mathbf{y}, s\rangle}, f$ is a fixed point of $P_{\langle\mathbf{y}, s\rangle} P_{\langle\mathbf{x}, s\rangle}$, and $e=P_{\langle\mathbf{x}, s\rangle} f$ (and thus $f=P_{\langle\mathbf{y}, s\rangle} e$ ). According to Theorem 4.3, $e$ is a critical point of $h(x, f)$ and $f$ is a critical point of $h(e, y)$. Without loss of generality, we may assume that $(S \mid S)=-1$. Since $h_{1}$ is constant on projective lines $\langle y, s\rangle$, it suffices to consider its values on the subspace $\langle s\rangle^{\perp}$ to find its critical points on $\langle\mathbf{y}, s\rangle$. Let $y \in\langle\mathbf{y}, s\rangle \cap\langle s\rangle^{\perp}$, so $\Delta(Y, S)=-(Y \mid Y)$. By Theorem 3.9, $\langle\mathbf{y}, s\rangle \cap\langle s\rangle^{\perp} \cap \Omega=\emptyset$, and $(Y \mid Y)>0$ and

$$
h_{1}(y)=-\frac{\Delta\left(Y-P_{\langle\mathbf{X}, S\rangle} Y\right)}{(Y \mid Y)}=-\left(1-\frac{\left.P_{\langle\mathbf{X}, S\rangle} Y \mid Y\right)}{(Y \mid Y)}\right) .
$$

The critical point $y$ satisfies the condition

$$
\frac{2\left(P_{\langle\mathbf{X}, S\rangle} Y \mid d Y\right)(Y \mid Y)-\left(P_{\langle\mathbf{X}, S\rangle} Y \mid Y\right)(Y \mid d Y)}{(Y \mid Y)^{2}}=0
$$

for all $d Y \in\langle\mathbf{Y}, S\rangle$. Without loss of generality, we can assume that $(Y \mid Y)=1$ so

$$
P_{\langle\mathbf{X}, S\rangle} Y=\left(P_{\langle\mathbf{X}, S\rangle} Y \mid Y\right) Y .
$$

Applying $P_{\langle\mathbf{X}, S\rangle}$ on both sides we obtain

$$
P_{\langle\mathbf{Y}, S\rangle} P_{\langle\mathbf{X}, S\rangle} Y=\left(P_{\langle\mathbf{X}, S\rangle} Y \mid Y\right) Y,
$$

which is true if and only if $Y$ is an eigenvector, $F$. So $f$ is a critical point oh $h_{1}(y)$ and $\widehat{f}$ is a critical point of its restriction to $\langle\mathbf{x}, s\rangle \cap \Omega$. Clearly, all critical points are of this kind.

(ii) Let $(S \mid S)=0$. In this case, the function $h_{1}(y)$ is defined on $\langle\mathbf{y}, s\rangle \backslash\langle s\rangle^{\perp}$ and constant on projective lines $\langle x, s\rangle$, and

$$
h_{1}(y)=\frac{(Y \mid Y)-\left(Y \mid P_{\langle\mathbf{X}, S\rangle} Y\right)}{(Y \mid S)^{2}} .
$$


So

$$
\begin{aligned}
d h_{1}= & 2 \frac{\left((d Y \mid Y)-\left(d Y \mid P_{\langle\mathbf{X}, S\rangle} Y\right)\right)(Y \mid S)^{2}}{(X \mid S)^{4}} \\
& -\frac{\left((Y \mid Y)-\left(Y \mid P_{\langle\mathbf{X}, S\rangle} Y\right)\right)(Y \mid S)(d Y \mid S)}{(X \mid S)^{4}} .
\end{aligned}
$$

The point $y$ is critical if

$$
\left(d Y \mid\left(Y-P_{\langle\mathbf{X}, S\rangle} Y\right)-\frac{S}{(Y \mid S)}\left(Y \mid Y-P_{\langle\mathbf{X}, S\rangle} Y\right)\right)=0,
$$

that is, if

$$
Y-P_{\langle\mathbf{X}, S\rangle} Y=\frac{S}{(Y \mid S)}\left(Y \mid Y-P_{\langle\mathbf{X}, S\rangle} Y\right) .
$$

Without loss of generality, we may assume that $(Y \mid S)=1$. After applying $P_{\langle\mathbf{Y}, S\rangle}$ and rearranging, we obtain

$$
P_{\langle\mathbf{Y}, S\rangle} P_{\langle\mathbf{X}, S\rangle} Y=Y-S\left(Y \mid Y-P_{\langle\mathbf{X}, S\rangle} Y\right) .
$$

The solution $Y$ of the equation is not an eigenvector. Let $U \in$ $\langle\mathbf{F}\rangle^{\perp}$. The projective line $\langle u, s\rangle$ is a fixed line of the $P_{\langle\mathbf{y}, s\rangle} P_{\langle\mathbf{x}, s\rangle}$. The line intersects the quadric in the unique critical point of $h_{1}(y)$ restricted to $\langle\mathbf{y}, s\rangle \cap \Omega$.

5.4. Two pencils in $\mathbb{R}^{3}$. In this section, we will focus on geometric cycles and pencils in $\mathbb{R}^{3}$.

Let $\langle\mathbf{x}, r\rangle \cap \Omega$ and $\langle\mathbf{y}, r\rangle \cap \Omega$ be two hyperbolic (Steiner) pencils, which determine two subcycles (circles or lines) in $\mathbb{R}^{3}$. Now $\langle\mathbf{x}\rangle=\left\langle x_{1}, x_{2}\right\rangle$ where $x_{1}$ and $x_{2}$ are proper cycles and similarly for $\langle\mathbf{y}\rangle=\left\langle y_{1}, y_{2}\right\rangle$. Since $(R \mid R)=-1$, the critical points of the function $h$ on $\langle\mathbf{x}, r\rangle \times\langle\mathbf{y}, r\rangle$ are of the form $(e, f)$, where $e$ and $f=P_{\langle\mathbf{y}, r\rangle}(e)$ are fixed points of the product of projectors. One of these critical points is $(r, r)$. We will be interested in the critical points on $\langle\mathbf{x}, r\rangle \cap \Omega \times\langle\mathbf{y}, r\rangle \cap \Omega$. Since $\langle\mathbf{x}, r\rangle \cap \Omega \times\langle\mathbf{y}, r\rangle \cap \Omega$ is a compact set, and $h$ is continuous, there must be two critical points $\left(\widehat{e}_{1}, \widehat{f}_{1}\right)$ and $\left(\widehat{e}_{2}, \widehat{f}_{2}\right)$ where the maximum and minimum are achieved.

Theorem 5.6. Let $\langle\mathbf{x}, r\rangle \cap \Omega$ and $\langle\mathbf{y}, r\rangle \cap \Omega$ be independent in the sense that they have no common cycles. The corresponding subcycles 
in $\mathbb{R}^{3}$ intersect if $\delta(\mathbf{x}, \mathbf{y}, r)=0$, they are linked if $\delta(\mathbf{x}, \mathbf{y}, r)<0$ and are nonlinked if $\delta(\mathbf{x}, \mathbf{y}, r)>0$. If $\delta(\mathbf{x}, \mathbf{y}, r)<0$, then the smallest and largest angle of intersection between a cycle $x \in\langle\mathbf{x}, r\rangle \cap \Omega$ and a cycle $y \in\langle\mathbf{y}, r\rangle \cap \Omega$ are reached at pairs $\left(\widehat{e}_{1}, \widehat{f}_{1}\right)$ and $\left(\widehat{e}_{2}, \widehat{f}_{2}\right)$, which are obtained by projecting the fixed points $e_{1}, e_{2}$ of $P_{\langle\mathbf{x}, r\rangle} P_{\langle\mathbf{y}, r\rangle}$ and $f_{1}=P_{\langle\mathbf{y}, r\rangle}\left(e_{1}\right), f_{2}=P_{\langle\mathbf{y}, r\rangle}\left(e_{2}\right)$ of $P_{\langle\mathbf{y}, r\rangle} P_{\langle\mathbf{x}, r\rangle}$ onto $\Omega$ along $r$.

Proof. The first statement follows directly from Proposition 5.2. Since $\Delta(\mathbf{X}, R)<0$ and $\Delta(\mathbf{Y}, R)<0$, the sign of $\delta(\mathbf{x}, \mathbf{y}, r)$ is equal to the sign of $\Delta(\mathbf{X}, \mathbf{Y}, S)$. Let $\left(E_{1}, E_{2}, R\right)$ and $\left(F_{1}, F_{2}, R\right)$ be bases of $\langle\mathbf{X}, R\rangle$ and $\langle\mathbf{Y}, R\rangle$ consist of eigenvectors of $P_{\langle\mathbf{X}, R\rangle} P_{\langle\mathbf{Y}, R\rangle}$ and $P_{\langle\mathbf{Y}, R\rangle} P_{\langle\mathbf{X}, R\rangle}$, respectively. Since the Lie form restricted to $\langle\mathbf{X}, R\rangle$ or $\langle\mathbf{Y}, R\rangle$ has index 1 and $(R \mid R)<0$, we can normalize them so that $\left(E_{i}, E_{j}\right)=\delta_{i j},\left(F_{i} \mid F_{j}\right)=\delta_{i j}$ and $\left(E_{i} \mid F_{j}\right)=\delta_{i j} \sqrt{\lambda_{i}}$, where $\lambda_{i}$ are corresponding eigenvalues (see subsection 5.2.1). Then,

$$
\Delta(\mathbf{X}, \mathbf{Y}, S)=-\left(1-\lambda_{1}\right)\left(1-\lambda_{2}\right) .
$$

If $\delta(\mathbf{x}, \mathbf{y}, r)>0$, then not all pairs of the cycles $(x, y), x \in\langle\mathbf{x}, r\rangle \cap \Omega$, $y \in\langle\mathbf{y}, r\rangle \cap \Omega$ intersect, which implies that the subcycles are nonlinked. If $\delta(\mathbf{x}, \mathbf{y}, r)<0$ then by Theorem 5.5, the extreme values of $h(x, y)=$ $-\sin ^{2} \alpha(x, y) \in\langle\mathbf{x}, r\rangle \cap \Omega \times\langle\mathbf{y}, r\rangle \cap \Omega$ are achieved at the two pairs $\left(\widehat{e}_{1}, \widehat{f}_{1}\right)$ and $\left(\widehat{e}_{2}, \widehat{f}_{2}\right)$ with maximal and minimal angles of intersection $\alpha$.

The situation with cones (and in general with $s$-pencils, where $(S \mid S)=0$ ) is different than in the case of subcycles (where $(S \mid S)<$ $0)$.

Theorem 5.7. Let $\langle\mathbf{x}, w\rangle \cap \Omega$ and $\langle\mathbf{y}, w\rangle \cap \Omega$ be cones.

(i) If $\delta(\mathbf{x}, \mathbf{y}, w)=0$, then two cones either share a common cycle or an oriented tangential plane or they have parallel axes.

(ii) If $\delta(\mathbf{x}, \mathbf{y}, w)<0$, then for any pair of cycles $(x, y) \in\langle\mathbf{x}, w\rangle \cap \Omega \times$ $\langle\mathbf{y}, w\rangle \cap \Omega$, their tangential distance exists. The minimal tangential distance is reached at a pair $(\widehat{e}, \widehat{f})$ which is obtained by projecting the fixed points $e$ of $P_{\langle\mathbf{x}, w\rangle} P_{\langle\mathbf{y}, w\rangle}$ and $f$ of $P_{\langle\mathbf{y}, w\rangle} P_{\langle\mathbf{x}, w\rangle}$ along $w$ onto $\Omega$. 


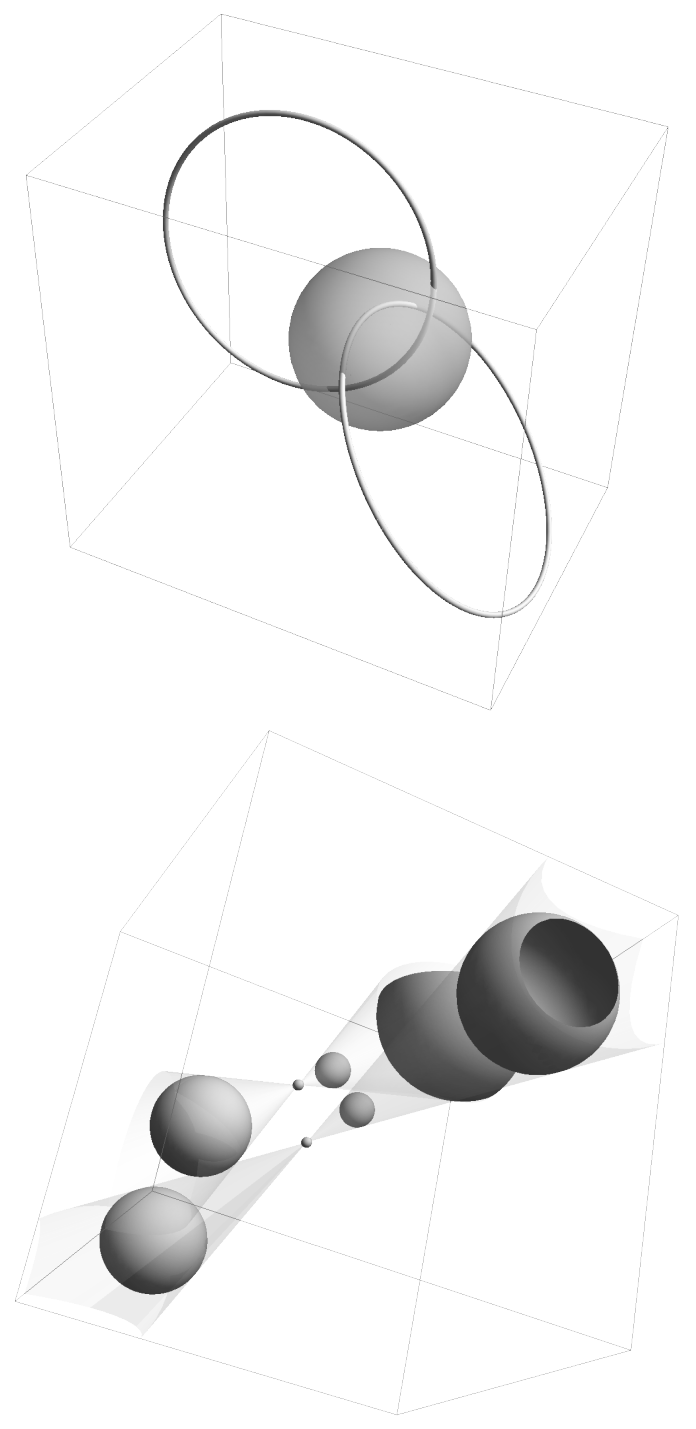

Figure 8. The case of two linked circles and two nonintersecting cones, $\delta<0$. 
(iii) If $\delta(\mathbf{x}, \mathbf{y}, w)>0$, then there exist two cycles, one of each family, which have no tangential distance.

Proof.

(i) The discriminant is equal to

$$
\delta(\mathbf{x}, \mathbf{y}, w)=-d^{2} \sin ^{2} \alpha .
$$

So the discriminant is zero either if $d=0$, that is, the minimal distance is zero and there is a common tangential plane, or $\alpha=0$ and axes of the cones are parallel. The discriminant is zero also if cycles in $(\mathbf{x}, \mathbf{y})$ are linearly dependent and the families share a common cycle, (see Proposition 5.2).

(ii) If the discriminant is negative, then the axes are not parallel and each pair of cycles containing a cycle from each family has a tangential distance, (see Proposition 5.5).

(iii) Follows from Proposition 5.5.

The meaning of the discriminant of two hyperbolic cones or subcycles can be expressed in terms of the special determinants, see subsection 3.5. Let us take a look at cone families. The discriminant

$$
\delta(\mathbf{x}, \mathbf{y}, w)=\frac{\Delta(\mathbf{X}, \mathbf{Y}, W)}{\Delta(\mathbf{X}, W) \Delta(\mathbf{Y}, W)}
$$

is proportional to the squared volume of the simplex spanned by tangential distances of cycles $\mathbf{x}$ and $\mathbf{y}$ divided by the product of the squared volumes of the facets spanned by tangential distances of $\mathbf{x}$ and y. In the case of two lines defined by a pair of points in space, the discriminant is equal to the squared volume of the simplex spanned by all four points divided by the product of squared distances between the points of the same line. It follows that

Proposition 5.8. The discriminant of two hyperbolic cone families is equal to zero if the volume of the contact simplex spanned by tangential distances is zero, is negative if the volume of the contact simplex is different from zero and is positive if there is no such simplex. 
In the case of subcycles the discriminant can be expressed in the terms of a quotient of volumes of simplices or polar sines, but the geometrical meaning is less evident.

Figure 8 on the top shows two linked subcycles corresponding to Steiner pencils $\langle\mathbf{x}, r\rangle \cap \Omega$ and $\langle\mathbf{y}, r\rangle \cap \Omega$. The sphere is the minimal sphere of the product family $\langle\mathbf{x}, \mathbf{y}, r\rangle \cap \Omega$. The circles intersect the sphere in antipodal points. On the bottom, the figure shows two nonintersecting cones. Two middle spheres are the spheres of minimal tangential distance.

\section{REFERENCES}

1. H. Behnke, Geometry, Fundamentals of mathematics, Volume 2, MIT Press, 1974.

2. T.E. Cecil, Lie sphere geometry with applications to minimal submanifolds, Springer, Berlin, 1992.

3. F. Ericson, The law of sines for tetrahedra and n-simplices, Geom. Ded. 7 (1978), 1-80.

4. J.P. Fillmore and A. Springer, Planar sections of the quadric of Lie cycles and their Euclidean interpretations, Geom. Ded. 55 (1995), 175-193.

5. P. Gritzmann and V. Klee, On complexity of some basic problems in computational convexity II, in Polytopes, abstract, convex and computational, Kluwer, Dordrecht, 1994.

6. B. Jurčič Zlobec and N. Mramor Kosta, Configurations of cycles and the Apollonius problem, Rocky Mountain J. Math. 31 (2001), 725-744.

7. , Geometric constructions on cycles, Rocky Mountain J. Math. 34 (2004), 1565-1585.

8. D. Kim, D.S. Kim and K. Sugihara, Apollonius tenth problem via radius adjustment and Moöbius transformation, Computer-Aided Design 38 (2006), 1421.

9. R.D. Knight, The Apollonius contact problem and Lie contact geometry, J. Geom. 83 (2005), 137-152.

10. S. Lie, Über Komplexe, inbesondere Linien- und Kugelkomplexe, mit Anwendung auf Theorie der partielle Differentialgleichungen, Math. Ann. 5 (1872), 145-208, 209-256 (Ges. Abh. 2, 1-121).

11. D. Pedoe, Geometry, Dover Publications, New York, 1988.

12. J.F. Rigby, The geometry of cycles, and generalized Laguerre inversion, in The geometric vein, The coxeter Festschrift, C. Davis, B. Grünbaum and F.A. Sherk, eds., Springer, New York, 1981.

13. I.M. Yaglom, On the circular transformations of Möbius, Laguerre, and Lie, in The geometric vein, The coxeter Festschrift, C. Davis, B. Grünbaum and F.A. Sherk, eds., Springer, New York, 1981. 
Department of Electrical Engineering, University of Ljubljana, Slovenia Email address: borut.jurcic@fe.uni-lj.si

Department of Computer and Information Science, University of Ljubljana and Institute of Mathematics, Physics and Mechanics, Ljubljana, Slovenia

Email address: neza.mramor@fri.uni-lj.si 